\title{
Comprehensive bioinformatic analysis reveals a cancer-associated fibroblast gene signature as a poor prognostic factor and potential therapeutic target in gastric cancer
}

\section{Cemre Ucaryilmaz-Metin}

Koc University Graduate School of Health Sciences

Gulnihal Ozcan ( $\square$ guozcan@ku.edu.tr)

Koc University School of Medicine

\section{Research Article}

Keywords: Gastric cancer, cancer-associated fibroblasts, extracellular matrix, tumor microenvironment, bioinformatics, prognostic biomarkers, therapeutic targets.

Posted Date: January 18th, 2022

DOI: https://doi.org/10.21203/rs.3.rs-1256927/v1

License: (c) (1) This work is licensed under a Creative Commons Attribution 4.0 International License. Read Full License 


\section{Abstract}

Background: Gastric cancer is one of the deadliest cancers in the world. The lack of prognostic markers and efficient molecular targets limits the success of current therapies. Therefore, we aimed to identify new prognostic markers and therapeutic targets in gastric cancer. Methods: We analyzed four GEO gene expression datasets with a network-based approach. We used The Cancer Genome Atlas (TCGA) stomach adenocarcinoma data as the validation set. Since all the hub genes indicated the cancerassociated fibroblast infiltration in gastric tumors, we performed a stepwise multivariate Cox regression analysis to identify a poor prognostic gene signature for cancer-associated fibroblast infiltration. Lastly, we searched the drug databases for drugs that target the signature genes. Results: Our network-based approach revealed the extracellular matrix components COL1A1, COL1A2, COL3A1, COL5A1, FN1, and SPARC as the central genes in gastric cancer. These genes were overexpressed and displayed poor prognostic significance in TCGA stomach adenocarcinoma samples. We demonstrated the substantial correlation between these genes and the cancer-associated fibroblast infiltration in gastric cancer. The stepwise multivariate Cox regression elucidated COL1A1 and COL5A1, together with ITGA4, Emilin1, and TSPAN9 as poor prognostic signature genes for the cancer-associated fibroblast infiltration. The search on drug databases revealed: collagenase clostridium histolyticum, ocriplasmin, and halofuginone which target collagen subunits; and natalizumab, firategrast, and BIO-1211 which target ITGA4 as the potential drugs for further investigation. Conclusions: Our study demonstrated the central role of extracellular matrix components secreted and remodeled by the cancer-associated fibroblasts in gastric cancer. The cancer-associated fibroblast gene signature we identified in this study carries a high potential as a predictive tool for poor prognosis in gastric cancer patients. Elucidating the mechanisms by which the signature genes contribute to poor patient outcomes can lead to the discovery of more potent moleculartargeted agents and; increase the therapeutic success in gastric cancer.

\section{Background}

Gastric cancer is the fifth most common cancer worldwide and the fourth leading cause of cancer-related deaths, the GLOBOCAN 2020 statistics report. More than one million people were diagnosed with gastric cancer, and more than 750,000 deaths occurred due to gastric cancer in 2020 (1). Stomach adenocarcinomas (STAD) constitute almost \% 95 of all gastric cancer cases. The mainstay of treatment in localized stomach adenocarcinoma is gastrectomy with total lymphadenectomy and chemotherapy (2). However, the tumors are commonly metastatic at the time of diagnosis. At this stage, complete resection is impossible, and currently available chemotherapeutics fail due to chemoresistance $(2,3)$.

Molecular-targeted agents against tumor-specific biomarkers and immunotherapy increased the treatment efficacy in certain cancers such as breast cancer, lung cancer, and melanoma (4). However, similar success is not valid in gastric cancer yet. Currently, molecular-targeted agents, nivolumab (antiPD-1), pembrolizumab (anti-PD-1), ramucirumab (anti-VEGFR2), and trastuzumab (anti-HER2), are approved in gastric cancer treatment. Unfortunately, they have limited efficacy on the overall survival of a 
limited group of advanced-stage patients with target positivity (5). New anti-cancer agents that target prognostic biomarkers are necessary for gastric cancer treatment.

In this study, we performed a comprehensive bioinformatic analysis to identify new prognostic markers, targetting which may increase the therapeutic efficacy in a large group of gastric cancer patients. We analyzed four gastric cancer gene expression datasets using a network-based approach

(Fig. 1 summarizes the steps followed in the study). Our analysis revealed an extracellular matrix (ECM) protein hub and implicated a central role for cancer-associated fibroblast (CAFs) in gastric tumors.

The CAFs are fibroblasts in the tumor microenvironment originating from activated fibroblasts and the endothelial or epithelial cells undergoing epithelial-mesenchymal transition (EMT). They secrete various ECM proteins and tumorigenic factors and remodel the ECM components to the advantage of cancer

cells. Emerging evidence demonstrates their pivotal roles in the progression of several cancers, including gastric cancer (6). Therefore, we further analyzed The Cancer Genome Atlas (TCGA) stomach

adenocarcinoma data and elucidated a poor prognostic gene signature for CAF infiltration using stepwise multivariate Cox regression. Lastly, we searched the drug databases to identify currently available drugs that may have therapeutic potential by targeting the signature genes we identified.

\section{Methods}

\section{Data collection and identification of differentially expressed genes}

We analyzed four GEO expression profiling datasets (GSE13911, GSE29272, GSE79973, GSE118916) (710), which used Affymetrix Human Genome or Gene Expression Arrays for profiling (https://www.ncbi.nlm.nih.gov/geo/). All four datasets bear a comparable number of samples from gastric cancer tissues and non-cancerous gastric tissues. Additional File 1: Table S1 lists the number of tissue samples and the profiling platforms at each dataset. In total, we analyzed 200 non-tumor gastric and 207 gastric tumor samples. To identify differentially expressed genes (DEGs) in gastric tumors compared to non-cancerous stomach samples, we used the GEO2R web tool (https://www.ncbi.nlm.nih.gov/geo/geo2r/). We applied log transformation and Benjamini \& Hochberg (False discovery rate) method to adjust the $p$-values ( $p$-value significance cut-off $=0,01$ ). The genes were filtered based on their log2-fold change $(\log F C)$ values. We accepted the genes with the log FC value $>1$ as the upregulated genes and with the log $\mathrm{FC}$ value $<-1$ as the downregulated genes. After identifying the DEGs at each dataset, we performed Venn Analysis to find DEGs common to all four datasets using the jvenn (an interactive Venn diagram viewer) (http://jvenn.toulouse.inra.fr/app/index.html).

\section{Functional annotation and enrichment analysis}

To perform functional enrichment and annotation clustering analysis of the DEGs, we used The Database for Annotation, Visualization, and Integrated Discovery (DAVID) (Version 6.8) (11) (https://david.ncifcrf.gov/). To understand the cellular compartments (GO-CC), molecular functions (GO$\mathrm{MF}$ ), and biological processes (GO-BP) at which the DEGs enriched, we performed gene ontology (GO) 
analysis. To understand the pathways at which the DEGs operate, we performed the Kyoto Encyclopedia of Genes and Genomes (KEGG) pathway analysis. The cut-off value for significance was chosen as $p<$ 0,05 . We analyzed gene lists for upregulated genes, downregulated genes, and all DEGs separately.

To compare the gene identities and gene ontologies enriched in different lists, we used Metascape (12) (https://metascape.org). To dissect the similarities and dissimilarities between gene lists, we analyzed the Circos plots, clustering dendrograms, network layouts for enriched gene ontologies, the proportion of the genes from different lists that fall into the same gene ontologies, and enrichment p-values.

\section{Protein-protein interaction network analysis}

To predict the potential connections and interactions of DEGs at their protein product level, we constructed the protein-protein interaction (PPI) network of the DEGs using The Search Tool for the Retrieval of Interacting Genes (STRING Version 11.0) (13) (https://string-db.org/). We utilized the minimum required interaction score as high confidence (0.7). We analyzed the resulting PPI network in Cytoscape (Version 3.8.2) to infer the topological parameters of each node (14) (https://cytoscape.org/). To investigate the network modules, we used Molecular Complex Detection (MCODE)- and; to construct a local network for the genes with the highest degree and their first neighbors, we used the Cytohubbaplugin at Cytoscape $(15,16)$.

\section{Gene expression profiling}

To confirm the differential expression of the hub genes in gastric cancer, we comparatively analyzed the gene expression profiles of 34 non-cancerous gastric tissues and 415 stomach adenocarcinoma samples in The Cancer Genome Atlas (TCGA) dataset using the UALCAN (University of Alabama Cancer Database) (17) (http://ualcan.path.uab.edu/). We also investigated the differential expression of the hub genes by tumor grade and stage (unpaired t-test was used for statistical analysis). To validate the results from the UALCAN, we analyzed The Genotype Tissue Expression Project (GTEx) data on GEPIA2 (18) (http://gepia2.cancer-pku.cn).

\section{Survival analysis}

To understand the impact of the genes on the survival of gastric cancer patients, we performed the Kaplan-Meier (KM) survival analysis of TCGA stomach adenocarcinoma samples on Tumor Immune Estimation Resource 2.0 (TIMER2.0) (19) (https://timer.cistrome.org). The stomach adenocarcinoma samples split into high or low expression groups based on the median expression level for each gene. Additionally, KM-Survival Curve for COL1A2 was extracted from the UALCAN to assess its prognostic role in gastric cancer. We generated the heatmaps that show the z-scores for each gene in distinct cancers using the "gene outcome" module in TIMER 2.0. We constituted the KM-survival curves for CAF infiltration that integrate gene expression data with the "immune association" tool in TIMER 2.0, which utilizes the log-rank test. Then we extracted the hazard ratios (HR), z-scores, and p-values from the multivariate Cox proportional hazard regression models built on TIMER 2.0. 


\section{Gene correlation analysis}

We investigated the correlation between the individual gene expression and CAF infiltration in different cancers by the "immune association" tool in TIMER 2.0. To examine the correlation between distinct genes, we used the "Gene Correlation" module in TIMER 2.0, which gives purity adjusted correlation coefficients calculated by partial spearman rank correlation. We extracted the correlation coefficients and the p-values from TIMER2.0. to generate a gene correlation heatmap on the Bioinfo Intelligent Cloud (BIC) imageGP tool (http://www.ehbio.com/Cloud_Platform/front/\#/). For hierarchical clustering of the heatmap, we selected the Spearman method on the imageGP.

\section{Potential drug search}

To identify potential drugs that interfere with the target genes or proteins, we searched the DrugBank (https://www.drugbank.com/) and the Drug-Gene Interaction database (DGIdb v4.2.0) (20) (https://www.dgidb.org/).

\section{Data Visualization}

To draw the bubble plots for enriched ontology terms, we used the BIC imageGP tool. To plot the gene expression profiles and hazards ratios for CAF infiltration and gene expression in different cancers, we used GraphPad Prism9.

\section{Results}

\section{Identification of differentially expressed genes in gastric cancer}

To identify the DEGs in gastric cancer vs. non-cancerous gastric tissues, we analyzed GSE13911, GSE29272, GSE79973, and GSE118916 datasets by GEO2R. Figure 2a shows the volcano plots and the number of the DEGs we detected in each dataset. The four datasets shared 83 DEGs: 38 upregulated-and 45 downregulated- genes (Fig. 2b-d; Additional File 1: Table S2).

\section{Functional annotation clustering and enrichment analysis of differentially expressed genes in gastric cancer}

To illuminate the biological functions and the pathways the DEGs enrich, we performed the functional annotation and enrichment analysis of 83 DEGs. The functional annotation clustering with the highest classification stringency in DAVID revealed 3 clusters (Table 1). The cluster with the highest enrichment score included the extracellular matrix-receptor interaction, focal adhesion, and PI3K-Akt signaling pathway.

Functional enrichment analysis of the upregulated genes indicated a key role for ECM organization, ECM remodeling, ECM-receptor interaction, and activation of pro-tumorigenic signaling pathways (Fig. 2e, Additional File 1: Table S3). The most enriched molecular functions were extracellular matrix structural 
constituent, platelet-derived growth factor binding and, integrin-binding. The top KEGG pathways related to the upregulated genes were ECM-receptor interaction, focal adhesion and, PI3K-Akt signaling (Fig. 2g, Additional File 1: Table S3). The downregulated genes enriched in metabolic processes and ion homeostasis (Fig. 2f-g, Additional File 1: Table S4).

\section{Protein-protein interaction network analysis and identification of the hub genes}

To investigate the potential interactions between the protein products of DEGs, we constructed a PPI network in STRING (Fig. 3a). The analysis of this network on Cytoscape 3.8.2. revealed two prominent hubs. The first hub was a larger-scale hub composed almost totally of ECM components. The top six proteins with the highest degree in this hub were: COL3A1 (collagen type III alpha 1 chain), FN1 (fibronectin 1), COL1A2 (collagen type I alpha 2 chain), COL1A1 (collagen type I alpha 1 chain), COL5A1 (collagen type $V$ alpha 1 chain), and SPARC (cysteine-rich acidic matrix-associated protein) respectively (Fig. 3b). Table 2 shows the topological parameters for these proteins. The topological parameters for the whole PPI network are listed in Additional File 1: Table S5. The components of the larger-scale hub were all upregulated genes in gastric tumors, except for COL2A1 (collagen type II alpha 1 chain) (Additional File 2: Fig. S1).

The second hub with the low clustering and connectivity was composed of metallothioneins MT1E, MT1F, MT1G, MT1H, MT1M, and MT1X (Fig. 3a-b), which are proteins responsible for cellular response to metal ions such as zinc and cadmium (21). This finding explains the GO-BP terms "cellular response to zinc ion" and "cellular response to cadmium ion" enriched in the list of downregulated genes (Fig. 2f). The interactions between these genes were based solely on co-expression and protein homology. All components of this hub were downregulated in gastric cancer (Additional File 2: Fig. S2).

\section{Analysis of the network modules}

To identify the individual modules in the PPI network, we used the MCODE tool in Cytoscape. MCODE identified three modules. The larger-scale ECM protein hub was represented by modules 1 and 2 and, the small-scale metallothionein hub was represented by module 3 in Fig. 3c. The 5 out of 6 highest degree nodes, COL1A1, COL1A2, COL3A1, COL5A1, and SPARC, were components of module 1, while the FN1 was in module 2.

Then we performed functional enrichment analysis to identify enriched KEGG pathways at each module. "ECM-receptor interaction" and "focal adhesion" were the common enriched KEGG pathways in modules 1 and 2 (Additional File 1: Table S6). "PI3K-Akt signaling pathway" was the third enriched pathway in module 1 . The only KEGG pathway detected in module 3 was mineral absorption. Hence the module analysis strengthened the connection of the six hub genes: COL1A1, COL1A2, COL3A1, COL5A1, FN1 and, $S P A R C$, with the 3 KEGG pathways: ECM-receptor interaction, focal adhesion and, PI3K-Akt signaling pathway, in gastric cancer. Since the clustering efficiency and connectivity were very low for the metallothionein module, we focused on the ECM genes in modules 1 and 2 for their potential as prognostic factors and therapeutic targets in gastric cancer. 
COL1A1, COL1A2, COL3A1, COL5A1, and FN1 are abundant structural proteins at the ECM. COL1A1 and COL1A2 are produced mainly by fibroblasts and together constitute the type I collagen in the connective tissue. COL3A1 and COL5A1 are the alpha-1 chains of type III and V collagen, which are found in connective tissue together with type I collagen $(22,23)$. FN1 is a glycoprotein involved in cell adhesion, wound healing, and metastasis. Besides their structural role, these proteins bind to the integrins on the cell membrane, and through focal adhesion kinases, they activate intracellular signaling pathways such as PI3K-Akt and MAPK pathways (24). SPARC encodes the cysteine-rich acidic matrix-associated protein that is an essential protein for ECM remodeling. It binds to collagens and fibronectin; and regulates the interactions of cells with the ECM (25).

We analyzed the first neighbors of these proteins in our PPI network using the Cytohubba tool in Cytoscape. All these proteins highly interacted with other structural ECM components: BGN (biglycan), THBS1/2 (thrombospondin 1/2), and VCAN (versican), or ECM remodeling enzymes like SERPINH1 (serpin family H member 1), SULF1 (sulfatase 1), and TIMP1 (tissue inhibitor matrix metalloproteinase 1) (Fig. 3d).

\section{Verifying the differential expression of six hub genes in gastric cancer}

To validate the differential expression of the six hub genes in gastric cancer, we analyzed the TCGA data in UALCAN. The expression of all six genes was significantly higher in stomach adenocarcinoma samples, compatible with our results (Fig. 4a). The significant upregulation of these genes in gastric cancer was also verified on GEPIA2 using gastric cancer data from the GTEx dataset (data not shown).

\section{Prognostic significance of the extracellular matrix hub genes in gastric cancer}

To investigate whether the six hub genes are involved in gastric cancer progression, we investigated their differential expression by tumor stage and grade on UALCAN using the TCGA data. For COL1A2, COL3A1, COL5A1, FN1, and SPARC, there was no significant upregulation in stage 1 patients compared to healthy controls (Fig. 4b). However, their expression was significantly higher in stage 2, 3, and 4 samples than the stage 1 samples. Only for $C O L 1 A 1$, the expression was high starting from stage 1 . After stage 2 , the increase in COL1A1 expression became much more prominent. These findings suggest that COL $1 A 1$ may be involved in both tumorigenesis and tumor progression. On the other hand, COL1A2, COL3A1, COL5A1, $F N 1$, and SPARC may be more involved in tumor progression from stage 1 to stage 2, at which the tumor cells gain the ability to invade surrounding tissues.

The expression of COL1A1, COL1A2, COL5A1, and SPARC was significantly high starting from grade 1 (Fig. 4c). The medians of expression for these genes gradually increased in grade 2 and 3 samples. For COL $3 A 1$ and $F N 1$, the upregulation in grade 1 disease compared to healthy gastric tissue was not statistically significant due to the high variance in grade1. However, their expression was significantly higher in grade 2 and grade 3 compared to grade 1 disease and normal tissue. These findings suggest that all six genes may be associated with a poorly differentiated phenotype in gastric cancer. 
Then we investigated the impact of upregulated COL1A1, COL 1A2, COL3A1, COL5A1, FN1, and SPARC on patient survival with KM-survival analysis (Fig. 4d). High expression of COL1A1, COL3A1, COL5A1, FN1, and $S P A R C$ was significantly associated with poor survival in gastric cancer patients $(\mathrm{p}<0.05)$. Although the survival curves for COL1A2 high vs. low expression samples were different, the difference was not significant enough to suggest that the high expression of $C O L 1 A 2$ is associated with poor survival $(p=0.0618)$. Despite that, the KM-survival curves for $C O L 1 A 2$ high vs. low/medium expression samples from the TCGA database were statistically different $(\mathrm{p}=0.029)$ (Additional File 2: Fig. S3). These findings support that $C O L 1 A 1, C O L 1 A 2, C O L 3 A 1, C O L 5 A 1, F N 1$, and SPARC are associated with poor prognosis in gastric cancer.

\section{The connection of the hub genes with the cancer-associated fibroblasts}

The CAFs are critical components at the cellular compartment of the tumor microenvironment that assemble and remodel the ECM. They secrete numerous ECM proteins, mainly fibrous collagens (type I, III, and $\mathrm{V}$ collagens) and fibronectin. They dynamically interact with the cells and signals in the tumor microenvironment; alter the ECM through matrix metalloproteinases (MMPs) which cleave the ECM components, and the lysyl oxidase (LOX) family enzymes which crosslink the collagens. The dynamic remodeling of ECM by CAFs facilitates cancer cell migration and invasion. Additionally, CAFs induce ECM stiffness in the tumor microenvironment, which is associated with poor survival in many cancers $(6,23)$.

All the six hub genes we detected are CAF markers in several cancers (26). Hence, our network-based analysis implicated a significant CAF infiltration in gastric cancer. Correlation analysis in TIMER2.0 revealed a statistically significant correlation for all six hub genes with CAF infiltration in stomach adenocarcinoma, supporting our hypothesis (Fig. 5). The correlation of these genes with the CAF infiltration was even higher than that of poor prognostic CAF signature genes recently identified in gastric cancer: THBS1, THBS2, INHBA (inhibin A), CXCL 12 (C-X-C motif chemokine ligand 12), $T G F B 2$ (transforming growth factor-beta 2), VEGFB (vascular endothelial growth factor B), COL 10A1 (collagen type X alpha 1 chain), AREG (amphiregulin) and EFNA5 (ephrin A5) (Additional File 2: Fig. S4) (27-29).

Then we investigated the correlation of the six hub genes with a list of CAF markers at the gene expression level in TCGA stomach adenocarcinoma samples. The CAF marker list included 27 genes; 18 commonly used CAF markers (highlighted blue in Fig. 6a) and 9 CAF-specific markers (highlighted red in Fig. 6a). We also included CXCL12, INHBA, THBS1, and THBS2 since they are CAF markers associated with an aggressive phenotype in gastric cancer $(27,28,30)$. In hierarchical clustering analysis of the correlation matrix, the six hub genes were highly correlated and clustered with $C O L 11 A 1$ (collagen type XI alpha1 chain), FAP (fibroblast activation protein), INHBA, MMP11 (matrix metalloproteinase 11), S100A4 (S100 calcium-binding protein A4) and THBS2 (Fig. 6a).

After that, we compared all components of the ECM protein hub with the CAF markers list in terms of identity and gene ontology in Metascape. Eight out of 38 upregulated genes in gastric cancer (ASPN, COL1A1, COL1A2, COL3A1, COL5A1, FAP, FN1, and SPARC) overlapped with CAF markers list (Fig. 6b, 
upper circos plot). Besides that, 28 out of 38 upregulated genes in gastric cancer and 23 out of $27 \mathrm{CAF}$ markers fell into the same ontology term that is statistically significantly enriched in both lists (Fig. 6b, lower circos plot). The two lists differed in terms of 3 main ontologies. The "peptide cross-linking" and "integrin $a_{4} \beta_{1}$ pathway" were enriched in the gastric cancer-upregulated gene list. The "mesenchyme development" was enriched in the CAF markers list (Fig. 6c). Network layouts for enriched ontology clusters given in Fig. 7a-c better demonstrate that the upregulated genes in gastric cancer highly overlap with the CAF markers in terms of gene ontologies. The most striking difference for upregulated genes in gastric cancer was the enrichment of the "integrin $a_{4} \beta_{1}$ pathway".

\section{Prognostic impact of the hub genes on gastric tumors with CAF infiltration}

CAFs display a heterogenous gene expression profile in the stroma of distinct cancers. Hence, they play anti-tumor or tumor-promoting roles depending on the tumor type (6). Studies suggest a tumor-promoting role for CAFs in gastric cancer $(29,31)$. Accordingly, KM-survival analysis on TCGA data showed that CAF infiltration is associated with poor survival with a hazard ratio of 5.24 in stomach adenocarcinoma (Table $3)$. Moreover, the outcome of CAF infiltration worsens with the increasing tumor stage in gastric cancer (Additional File 2: Fig. S5).

Then, we investigated whether high expression of the six hub genes further worsens the prognosis in gastric tumors with CAF infiltration. We performed Cox proportional hazard regression analysis that considers both gene expression profiles and CAF infiltration in the TCGA stomach adenocarcinoma samples. Out of the six genes, the expression of $C O L 1 A 1, C O L 1 A 2, C O L 3 A 1$, or COL5A1 increased the zscore and hazards ratio for CAF infiltration (Table 3). The high COL5A1 expression led to the highest increase in the risk for poor survival $(z=2.666, H R=8.584)$. The high FN1 or SPARC expression did not increase the $z$-score and hazards ratio for CAF infiltration.

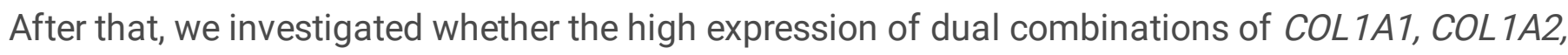
COL $3 A 1$, or COL5A1 exacerbate the outcome of CAF infiltration in stomach adenocarcinoma (Table 3). Concomitant high expression of $C O L 1 A 1$ and $C O L 5 A 1$ increased the hazard ratio most for CAF infiltration in TCGA samples $(z=2.924, \mathrm{HR}=11.654)$. The hazard ratio of CAF infiltration with this dual gene combination was even higher than that with the quadruple combination of collagen subunits. We also investigated the impact of CAF markers highly clustered with COL $1 A 1$ and COL5A1 in correlation analysis, namely THBS2, FAP, INHBA, S100A4, COL11A1, or MMP11, on the outcome of CAF infiltration in stomach adenocarcinoma. Except for MMP11, all slightly increased the hazard ratio for CAF infiltration (Additional File 1: Table S7).

Recently, Grunberg et. al suggested THBS1, THBS2, and INHBA; and Liu et. al suggested TGFB2, VEGFB, COL 10A1, AREG, and EFNA5 as poor prognostic signatures for CAF infiltration in gastric cancer. To compare the prognostic significance of these two gene signatures with that of COL 1A1 and COL5A1, we investigated the Cox regression models for these two signatures. The z-scores and hazard ratios for both signatures were lower than those for COL $1 A 1$ and $\operatorname{COL5A1}$ (Additional File 1: Table S8). These findings 
suggested a high potential for $C O L 1 A 1$ and $C O L 5 A 1$ as a poor prognostic signature in CAF infiltrated gastric tumors.

\section{Prognostic significance of COL1A1 and COL5A1 for CAF infiltration in other cancers}

At the next step, we asked whether COL1A1 and COL5A1 increase the poor prognostic impact of CAF infiltration in other cancers with a poor outcome profile for the six hub genes like gastric cancer. We investigated the risk scores for COL1A1, COL1A2, COL3A1, COL5A1, FN1, or SPARC at each tumor type in the TCGA dataset. We detected four cancer types with increased risk scores for all six genes: adrenocortical carcinoma (ACC), bladder urothelial carcinoma (BLCA), kidney renal papillary cell carcinoma (KIRP), and mesothelioma (MESO) (Fig. 8a). High CAF infiltration was also associated with an increased risk score in all four cancers (Fig. 8b). Despite that, the addition of COL 1A1 and COL5A1 to the Cox model led to a decreased risk score for CAF infiltration in adrenocortical carcinoma, kidney renal papillary cell carcinoma, and mesothelioma (Fig. 8c).

Identifying the players for the opposing roles of $C O L 1 A 1$ and $C O L 5 A 1$ in different cancers could reveal new insights into the field. To predict possible players, we extracted the list of genes that correlate with the expression of $C O L 1 A 1$ and $C O L 5 A 1$ in adrenocortical carcinoma, kidney renal papillary cell carcinoma, and mesothelioma. We identified 25 genes that are highly correlated (rho $\geq 0.5$ ) with both COL $1 A 1$ and COL5A1 in all three cancers (Fig. 8d). Then we comparatively analyzed this list with the list of genes upregulated in gastric cancer. The two lists shared seven genes (Fig. 8e, upper circos plot). The 18 genes common to the three cancers fell into the same gene ontology as the 27 genes upregulated in gastric cancer (Fig. 8e, lower circos plot). Despite these overlaps, more than ten ontologies are differentially enriched in the list of upregulated genes in gastric cancer (Fig. 8f). Among these, "integrin $a_{4} \beta_{1}$ pathway" and "peptide crosslinking" were striking, since they were the two ontologies that were enriched at the upregulated gene list in gastric cancer compared to the CAF markers list (Fig. $6 \mathrm{c}$ and 7ac). This observation emphasized the role of "integrin $a_{4} \beta_{1}$ pathway" and "peptide crosslinking" together with CAF infiltration in gastric cancer.

\section{Contribution of Integrin a4 $\beta 1$ pathway to the poor prognostic impact of COL1A1, COL5A1, and CAF infiltration in stomach adenocarcinoma}

Integrins are heterodimeric transmembrane proteins that are involved in cell-cell or cell-ECM adhesions. They bind ECM components, mainly collagen, and fibronectin, activate intracellular signaling pathways, and regulate cell survival, proliferation, migration, and differentiation. Integrin $\alpha_{4} \beta_{1}$ is a heterodimer of integrin $a_{4}$ (ITGA4) and integrin $\beta_{1}$ (ITGB1). ITGB1 couples with a large variety of $a$ integrin subunits (32). However, ITGA4 couples with integrin $\beta_{1}$ or $\beta_{7}$ subunits $(33,34)$.

The integrin $a_{4} \beta_{1}$, also known as very late antigen-4 (VLA-4), is expressed on various immune cells, mediating the migration of leukocytes to the inflammatory sites via interaction with VCAM-1 (vascular cell adhesion protein 1) (33). Additionally, it binds to ECM components and takes part in fibronectin 
assembly (35). Increased expression of $a_{4} \beta_{1}$ integrin is associated with tumor progression and chemoresistance in cancer. The interaction of integrin $a_{4} \beta_{1}$ on the tumor cell membrane with the VCAM-1 on vascular endothelial cells is involved in metastasis (33). The interaction of $a_{4} \beta_{1}$ integrin with fibronectin suppressed apoptosis via FAK-mediated suppression of $\mathrm{p} 53$, and PI3K/Akt mediated upregulation of $\mathrm{Bcl}-2$ in myeloma cells. Increased integrin $\mathrm{a}_{4} \beta_{1}$ expression was associated with increased binding of melanoma cells to collagen I and collagen IV, and invasion through fibronectin (36). Moreover, $a_{4}$ integrin was suggested to affect a drug efflux mechanism independent from its coupling with $\beta_{1}$ integrin (37). However, the mechanisms by which integrin $a_{4} \beta_{1}$ heterodimer or $a_{4}$ integrin monomer contribute to invasion, metastasis, and chemoresistance in cancer are not exactly known.

To understand whether the integrin $a_{4} \beta_{1}$ potentiates the poor prognostic impact of $C A F s$, we analyzed the Cox regression model for CAF infiltration that considers the expression of ITGA4, ITGB1, or both in addition to COLIA1 and COL5A1. The addition of ITGA4 to the model increased the hazard ratio and zscore in stomach adenocarcinoma $(z=2.963, \mathrm{HR}=12.247)$ (Table 4). However, only ITGB1 or ITGA4 and ITGB1 slightly decreased the hazard ratio and z-score, which may be due to a less selective coupling of ITGB1 with several integrin a subtypes (Additional File 1: Table S9). These findings supported that ITGA4 may worsen the poor prognostic impact of CAFs in gastric cancer.

ITGA4 interacts with signaling molecules, receptors, and kinases that take part in ECM organization, integrin signaling, and cell-matrix adhesion (Additional File 2: Fig. S6A-B). Among the integrin $a_{4} \beta_{1}$ partners, FN1, osteopontin (secreted phosphoprotein1: SPP1), THBS1, and EMILIN-1 (Elastin microfibril interface-located protein 1) are ECM proteins; JAM2 (junctional adhesion molecule 2), JAM3 (junctional adhesion molecule 3), MADCAM1 (mucosal vascular addressin cell adhesion molecule 1), and VCAM1 are membrane-bound proteins. Their interaction with integrin a4 $\beta 1$ makes them potential players for the poor prognostic impact of ITGA4 $(35,38-41)$. We asked whether $F N 1$, one of the six hub genes we detected in network analysis, strengthen the poor prognostic effect of ITGA4 on CAF infiltration. However, the addition of $F N 1$ to the COL1A1, COL5A1, and ITG4 Cox regression model decreased the poor prognostic impact of CAF in stomach adenocarcinoma (Additional File 1: Table S10). THBS1 acted similarly and decreased the hazard ratio. JAM2, JAM3, MADCAM1, SPP1, and VCAM1 slightly increased the hazard ratio. On the other hand, Emilin1 substantially increased the poor prognostic impact of CAFs in the COL1A1, COL5A1, and ITG4 model, raising the hazard ratio from 12.247 to 28.315 (Table 4).

The EMILIN-1 is a member of the elastin microfibrillar interface proteins (EMILINs) family, expressed as a homotrimer at the ECM (42). Since fibroblasts are the major sources of EMILIN-1 at the ECM, it is accepted as a fibroblast marker (26). The interaction of EMILIN-1 with integrin $\alpha_{4} \beta_{1}$ is involved in cell adhesion and migration (43). The increase in the poor prognostic impact of CAFs in stomach adenocarcinoma with the addition of Emilin 1 as a covariate to the Cox model was surprising since EMILIN-1 is known as a tumor suppressor that exerts anti-proliferative action via integrin $\alpha_{4} \beta_{1}$ in cell and in vivo models $(42,44)$. Despite that, some reports suggest a pro-tumorigenic role for EMILIN-1 in ovarian serous tumors and osteosarcoma $(45,46)$. 
A recent study reported that the action of EMILIN-1 to inhibit the MAPK pathway and suppress proliferation in gastric cancer cells might depend on Tetraspanin9 (TSPAN9) (47), which is a member of tetraspanin family membrane receptors with four transmembrane domains. These receptors are involved in signal transduction, cell adhesion, invasion, and migration. TSPAN9 is alluded to have anti-cancer effects in gastric cancer, suppressing proliferation, invasion, and migration in gastric cancer cell lines (48, 49). Despite that, adding TSPAN9 to the COL1A1, COL5A1, ITGA4, and Emilin 1 Cox model further increased the hazard ratio to 36.813 for CAF infiltration in stomach adenocarcinoma samples (Fig. 9a, Table 4). However, the hazard ratios remained zero with the stepwise addition of ITGA4, Emilin1, and TSPAN9 to the Cox model in adrenocortical carcinoma, kidney renal papillary cell carcinoma, and mesothelioma (Table 4). All this data suggested COL 1A1, COL5A1, ITGA4, Emilin1, and TSPAN9 as a poor prognostic CAF signature with high specificity to stomach adenocarcinoma.

We further investigated the expression profiles of the signature genes and their correlation with CAF infiltration (Fig. 9b-e). Mesothelioma and stomach adenocarcinoma displayed a higher expression profile for the COL1A1, COL5A1, ITGA4, and Emilin1, in comparison to adrenocortical carcinoma and kidney renal papillary cell carcinoma. The expression of TSPAN9 was similar in all four cancers (Fig. 9b). Correlation between the COL $1 A 1$ or COL5A1 expressions and CAF infiltration was strong in all four cancers (rho $>0.5$ ). ITGA4 expression poorly correlated with the CAF infiltration, but the correlation was slightly higher in kidney renal papillary cell carcinoma and stomach adenocarcinoma. Strikingly, the correlation of Emilin 1 and TSPAN9 with CAF infiltration was quite strong in stomach adenocarcinoma compared to a poorer correlation in the other three cancers (Fig. 9c).

Further investigation revealed that the expression of ITGA4 increases with stage in stomach adenocarcinoma (Fig. 9d). Although there was a significant decrease in Emilin1 and TSPAN9 levels in stage 1 compared to healthy stomach tissue, their expression increased again at stage 2 , reaching the level of or above that of normal tissues (Fig. 9e-f). A similar pattern was not observed for adrenocortical carcinoma, kidney renal papillary cell carcinoma, and mesothelioma (Additional File 2: Fig. S7).

The KM-survival analysis did not indicate a prognostic role for ITGA4, Emilin1, or TSPAN9 in stomach adenocarcinoma per se (Additional File 2: Fig. S8A-C). However, their hazard ratios increased with the stage (Additional File 2: Fig. S8D-F). This was in parallel to the increase in the poor prognostic impact of CAF infiltration by stage in stomach adenocarcinoma (Additional File 2: Fig. S5), suggesting a stage and CAF dependent role for ITGA4, Emilin1, and TSPAN9.

\section{Search on drugs that target COL1A1, COL5A1, and ITGA4}

Lastly, we searched for currently available drugs that target COL1A1, COL5A1, and ITGA4. Our search on DrugBank and DGIB revealed three agents which target COL1A1 and COL5A1: collagenase clostridium histolyticum, halofuginone, and ocriplasmin; and three agents which target ITGA4: natalizumab, firategrast, and BIO-1211 (Fig. 10). 
Collagenase clostridium histolyticum and ocriplasmin are enzymes that cleave COL1A1 and COL5A1. They also have proteolytic activity on COL3A1 and FN1, respectively $(50,51)$. Collagenase clostridium histolyticum is used on skin ulcers to hasten wound healing and Dupuytrens' disease to resolve contractures by digesting collagen $(52,53)$. Intra-tumoral or intravenous injection of collagenase increased the diffusion of large drug molecules in tumor models (54). Intraperitoneal administration of collagenase was reported to increase the efficacy of chemotherapy by cleaving the tumor stroma in a rat model of colorectal cancer peritoneal metastasis (55). Ocriplasmin is used to remove adhesions in symptomatic vitreomacular adhesion (51). Like our study, another bioinformatics study suggested ocriplasmin as a potential anti-cancer agent (56). But, to the best of our knowledge, ocriplasmin has not been tested in cancer before.

Halofuginone is an alkaloid that suppresses the expression of the COL1A1 gene, cell migration, and ECM formation. Besides its' antifibrotic and anti-angiogenetic actions, halofuginone shows antiproliferative effects by inhibiting TGF $\beta /$ Smad3 signaling $(57,58)$. Halofuginone, showed an apoptotic effect in prostate cancer and Wilms' tumor cells by inhibiting the transformation of fibroblasts to myofibroblasts (59), which carry similar features with CAFs (6). Halofuginone also acted synergistically with gemcitabine and suppressed tumorigenesis in a mouse pancreatic cancer model by reducing the number of stromal myofibroblasts and generation of ECM (60).

Integrin $\alpha_{4} \beta_{1}$ is a significant therapeutic target in chronic inflammatory diseases and cancer. Natalizumab, the monoclonal antibody against integrin $a_{4}$ subtype, was approved in multiple sclerosis and inflammatory bowel disease. However, its long-term use is associated with progressive multifocal leukoencephalopathy (41). Although abrilumab and vedolizumab are listed as integrin $\mathrm{a}_{4}$ targeting agents, their action is specific to integrin $a_{4} \beta_{7}$ heterodimer $(61,62)$. Besides monoclonal antibodies, small molecule inhibitors that target integrin $\alpha_{4}$ such as firategrast and BIO-1211 are available $(63,64)$. To the best of our knowledge, these agents have not been tested for their therapeutic efficacy in cancer yet.

\section{Discussion}

In this study, we performed a comprehensive bioinformatic analysis to identify new biomarkers targeting which may have a therapeutic potential in a large group of gastric cancer patients. Our network-based approach revealed an upregulated ECM protein hub where the COL1A1, COL1A2, COL3A1, COL5A1, FN1, and $S P A R C$ were the most central genes. High expression of all these genes was associated with tumor progression and decreased survival in gastric cancer patients. Further analysis demonstrated that the upregulated genes in gastric cancer were highly correlated with CAF infiltration. We performed stepwise multivariate Cox regression analysis and identified COL 1A1, COL5A1, ITGA4, Emilin 1, and TSPAN9 as interacting covariates, which potentiated the poor prognostic impact of CAF infiltration specifically in stomach adenocarcinoma. These findings emphasize the key role of the tumor microenvironment in gastric cancer. 
Tumor cells are in a dynamic interaction with their microenvironment that consists of an ECM compartment and a cellular compartment. They orchestrate both compartments for their advantage to facilitate tumor progression, invasion, and metastasis. By remodeling the ECM, tumor cells form a protective barrier against immune surveillance and the diffusion of anti-cancer agents. In addition to this physical protection, tumor cells alter the composition of ECM and potentiate pro-tumorigenic intracellular signaling pathways via binding of ECM components to transmembrane receptors - mainly integrins. Interaction of collagens, fibronectin, and other ECM components with integrins activates focal adhesion kinase (FAK), leading to activation of Src, Wnt, PI3K-Akt, and MAPK signaling pathways (65, 66). Accordingly, the functional enrichment analysis in this study demonstrated the key role of ECMreceptor interaction, focal adhesion, and PI3K-Akt activation in gastric cancer (Table 1 and Additional File 1: Table S6).

There is a great interest in the use of ECM components as biomarkers in various cancers $(67,68)$. Our study identified the six ECM genes as prognostic biomarkers in gastric cancer. Different bioinformatics and experimental studies support our findings. Zhao et al. detected upregulation of $C O L 1 A 1$, COL1A2, COL3A1, COL5A1, and FN1 in gastric cancer patient samples. COL1A1 was suggested as an early diagnostic marker for gastric cancer since it was upregulated in pre-malignant lesions (69). Li et al. detected upregulation of both $C O L 1 A 1$ and $C O L 1 A 2$ in gastric tumors compared to normal and premalignant tissues, whereas they detected only the upregulation of $C O L 1 A 1$ in premalignant tissues compared to normal tissue. COL1A2 expression was correlated with the tumor size and invasion depth in the study (70). Similarly in our analysis of TCGA data COL $1 A 1$ expression was higher starting from stage 1 stomach adenocarcinoma, but $C O L 1 A 2$ expression increased after stage 2 compared to normal gastric tissue. These findings suggest the involvement of COL1A1 in gastric carcinogenesis, while the COL1A2 may be more involved in tumor progression. Wang et al. built a seven gene signature, which included $C O L 1 A 1, C O L 1 A 2$, and SPARC, associated with poor prognosis in gastric cancer (71). FN1 was detected as one of the six poor prognostic genes in gastric cancer in another study (72).

The key findings that make this study different from other studies on biomarker identification are the association demonstrated between the hub genes and CAF infiltration in gastric cancer and the identification of a poor prognostic signature for CAFs. CAFs can exhibit a pro-tumorigenic or antitumorigenic role in a tumor-dependent manner. Our study and others' findings support a poor prognostic role for CAFs in gastric cancer $(29,31)$ (Table 3 and Additional File 2: Fig. S5). Zeng et. al. computationally analyzed the cell infiltration pattern in the tumor microenvironment of 1,524 gastric cancer patients. The fibroblast infiltration was the greatest risk factor in tumor microenvironment phenotype with the poorest overall survival (73). Zhou. et al. observed increased deposition, alignment, length, and straightness of collagen, the abundance of fibroblasts, and increased expression of collagen cross-linking enzyme lysyl oxidase-like 2 (LOXL2) at the stroma of gastric cancer specimens. Increased collagen width was the most significant predictor of poor overall survival in two independent gastric cohorts in the study (74). Accordingly, peptide cross-linking was one of the enriched gene ontologies in our upregulated gene list in gastric cancer. These findings point out the poor prognostic significance of collagen deposition and remodeling by CAFs in gastric cancer. 
Based on the strong association we observed between collagen subunits and CAF infiltration in gastric cancer, we investigated their impact on the poor prognostic role of CAFs in gastric cancer. We identified COL $1 A 1$ and COL5A1 as poor prognostic factors for CAF infiltration (Table 3). The opposite effect of this gene signature in other cancers (Fig. 8e) led us to identify ITGA4, Emilin1, and TSPAN9 as interactors that potentiate the poor prognostic effect of CAFs further in gastric cancer.

The delineation of ITGA4 as a poor prognostic factor was not surprising since increased expression of integrin $a_{4} \beta_{1}$ is associated with tumor progression (33). However, the poor prognostic effect of Emilin 1 and TSPAN9 was surprising since EMILIN-1 is regarded as a tumor suppressor which acts synergistically with TSPAN9. Knockout of Emilin1 or suppression of EMILIN-1 - integrin $a_{4} \beta_{1}$ interaction was associated with decreased expression of the tumor suppressor PTEN, and increased activity of PI3K/Akt and ERK1/2 pathways, leading to hyperproliferation of dermal fibroblasts and keratinocytes, increased skin carcinogenesis and lymph node metastasis $(42,44)$. Knocking out Emilin 1 or transgenic expression of an Emilin 1 mutant with impaired binding to integrin $\alpha_{4} \beta_{1}$ increased the susceptibility of mice to develop colon cancer (75). Based on these findings Emilin 1 was proposed as a tumor suppressor. However, Emilin 1 overexpression was detected in serous ovarian carcinoma, soft tissue osteosarcoma, and lowgrade glioma (LGG) which are malignant tumors with high recurrence rates $(45,46,76)$. This suggests a tissue-dependent anti-tumorigenic or pro-tumorigenic role for EMILIN-1.

Recently, EMILIN-1 was suggested to increase TSPAN9 expression in gastric cancer cell lines and form a complex with TSPAN9 to synergistically inhibit FAK/Ras/Erk pathway and suppress invasion and migration. Since overexpression of only Emilin1 did not induce a similar anti-tumor response in gastric cancer cell lines, it was suggested that the anti-tumor effect of EMILIN-1 may be dependent on TSPAN9 (47). Therefore, we tested whether the addition of TSPAN9 to the CO1A1, COL5A1, ITGA4, and Emilin1 Cox model can decrease the poor prognostic impact of CAF infiltration in gastric cancer. Unexpectedly, the hazard ratio and risk score for the CAF infiltration increased further in stomach adenocarcinoma but remained zero in adrenocortical carcinoma, kidney renal papillary cell carcinoma, and mesothelioma (Table 4).

Although some studies suggest an anti-cancer role for TSPAN9 in gastric cancer cell lines $(48,49)$, high TSPAN9 expression was associated with resistance to 5-fluorouracil via suppressing autophagy (77). Expression of TSPAN9 was significantly lower in gastric cancer tissue compared to adjacent normal gastric tissue in a cohort of 105 gastric cancer samples. However, the same cohort reported high TSPAN9 expression as a poor prognostic factor on survival (78). Therefore, whether EMILIN1 and TSPAN9 exert an anti-tumor effect or pro-tumorigenic effect in gastric cancer is not clear yet.

Our investigation on TCGA stomach adenocarcinoma data does not suggest either a poor or a good prognostic role for Emilin1 or TSPAN9 per se (Additional File 2: Fig. S8B-C). Despite that, the Emilin1 and TSPAN9 displayed a stage-dependent increase in expression (Fig. 8f) and their hazard ratios increased by stage in parallel to the stage-dependent increase in the hazard ratio of CAFs (Additional File 2: Fig. S8EF). Moreover, stepwise addition of these two genes as covariates to the CO1A1, COL5A1, and ITGA4

Page $15 / 43$ 
multivariate Cox model tremendously increased the hazard ratio for the poor prognostic impact of CAF infiltration, which suggested a stage and CAF dependent role for Emilin 1 and TSPAN9 in gastric cancer (Table 4). This may explain why these two genes display anti-tumor effects in monoculture gastric cancer cell lines but display poor prognostic effects in the gastric cancer patient cohort by Feng et al. (78) and TCGA stomach adenocarcinoma cohort we analyzed in this study.

It may be speculated that the ECM remodeling enzymes secreted from CAFs may cleave EMILIN-1 and prevent the anti-tumor action of EMILIN-1 together with TSPAN9 despite their high expression at the tumor tissue. Accordingly, proteolytic cleavage of EMILIN-1 by MMPs or neutrophil elastase was suggested as a mechanism for pro-tumorigenic effect in some tumors with high Emilin 1 expression (40, 79,80 ). For ECM proteins, it is also not unusual to serve different functions in cleaved forms vs. multimeric forms (81). A similar mechanism may explain the context-dependent role of EMILIN-1 and TSPAN9 in gastric cancer. Although there is no evidence in the literature yet for multimerization of EMILIN-1, this may also be a possible mechanism for its context-dependent action, since our analysis pointed out "protein-crosslinking" as an enriched biological process in gastric cancer. Elucidation of these molecular and cellular mechanisms may present EMILIN-1 and TSPAN9 as new therapeutic targets in gastric cancer. This will be addressed in our future studies.

Besides bringing about new research questions, answering of which may offer new therapeutic strategies in gastric cancer, our study put forth a multivariate Cox proportional hazard model to predict the poor prognostic impact of CAF infiltration in gastric cancer. Recently, Grunberg et. al suggested THBS1, THBS2, and INHBA; and Liu et. al suggested TGFB2, VEGFB, COL1OA1, AREG, and EFNA5 as poor prognostic signatures for $C A F$ infiltration in gastric cancer $(27,29)$. We compared the prognostic impact of these two signatures on CAF infiltration with that of COL1A1 and COL5A1. The z-score and hazard ratio for the COL1A1 and COL5A1 combination was higher compared to both signatures (Table 4, Additional File 1: Fig. S8). The hazard ratio of CAF infiltration in COL1A1, COL5A1, ITGA4, Emilin1, and TSPAN9 signature was even much higher (Table 4), presenting a new poor prognostic signature for CAFs with high potential in gastric cancer. One possible advantage of THBS1, THBS2, and INHBA signature may be its predictive ability in liquid biopsies since the signature genes are secreted through extracellular vesicles (27). Further studies are needed to assess and compare the predictive potential of all these signatures in different biopsy specimens.

To build the poor prognostic gene signature for CAF infiltration, we analyzed the TCGA stomach adenocarcinoma data in TIMER 2.0. TIMER 2.0 implements four different algorithms, namely EPIC, MCPCounter (Microenvironment Cell Populations-counter), Xcell, and TIDE (Tumor Immune Dysfunction and Exclusion) to predict the relative proportions of different cell populations in tumor samples (82-85). All these algorithms device reference gene expression profiles for each cell type, established from the RNAseq profiles of circulating immune cells and non-cancerous cells that infiltrate the tumors. One limitation to the study may be that only the TIDE algorithm predicted a poor prognostic impact for CAF infiltration in COL 1A1, COL5A1, ITGA4, Emilin1, and TSPAN9 multivariate Cox model in gastric cancer. One reason may be that the reference gene expression profiles for CAFs in all four algorithms are different, which leads to 
differences in the allocation of samples to high vs. low CAF infiltration groups. Dissecting these differences in detail may improve the power of these algorithms to predict the abundance of CAFs in tumor samples. Moreover, mostly melanoma samples are used to establish reference gene expression profiles for tumor-infiltrating cells. These reference gene expression profiles may not exactly reflect the gene expression pattern for CAFs in gastric cancer or other cancers. Besides intertumoral heterogeneity, intra-tumoral heterogeneity in CAFs further complicates the picture (6). Building tumor and subclonespecific reference gene expression profiles for CAFs may better illuminate the prognostic role of CAFs and their interactor genes in cancer. Such an approach may also reveal new molecular targets to prevent CAF infiltration in cancer.

Among the signature genes we identified in this study, targeting the COL1A1, COL5A1 and ITGA4 may have a therapeutic potential in gastric tumors with high CAF infiltration. Our search in drug databases brings out collagenase clostridium histolyticum, halofuginone, and ocriplasmin as agents that act on COL1A1 and COL5A1. Their anti-cancer action should be validated first in gastric cancer cell models and in vivo studies. Among these three, halofuginone seems to be the closest candidate for use as an anticancer agent in gastric cancer since it showed promising anti-cancer effects in other cancers. It may also prevent the formation of CAFs $(59,60)$. Moreover, collagenase clostridium histolyticum and ocriplasmin carry risks, since cleavage of collagens may lead to a release of several growth factors that induce tumor progression. Additionally, their systemic use can be problematic in cancer due to the risk of organ and vascular toxicity (54). Active targeting to the gastric tumors via nanocarriers or local administration may allow their use in gastric cancer. But still, there may be destructive effects in healthy gastric tissue limiting the doses that could be used in patients safely. All these points should be addressed in future studies.

Integrin $a_{4} \beta_{1}$ also has potential as a therapeutic target in cancer since it has a key role in metastasis, chemoresistance, angiogenesis, and lymphangiogenesis (33). Despite that, agents targeting integrin $a_{4} \beta_{1}$ have not entered the clinical trials for cancer. The concerns about the risk of cancer development with targeting integrin $a_{4} \beta_{1}$ may be the reason since this action inhibits the migration of lymphocytes. However, the comparative analysis did not reveal an increased risk of cancer with natalizumab (86). Uncovering the signaling mechanisms by which integrin $a_{4} \beta_{1}$ contribute to cancer may lead to the development of new strategies for targeting integrin $\alpha_{4} \beta_{1}$ without raising the concerns about cancer development.

\section{Conclusion}

In this study, we identified the key role of an upregulated ECM protein hub and CAFs in gastric cancer. The most central genes in the ECM hub, namely COL 1A1, COL1A2, COL3A1, COL5A1, FN1, and SPARC were all $\mathrm{CAF}$ markers that can be used as prognostic biomarkers in gastric cancer. Besides secretion of the key ECM proteins, CAFs may further worsen the prognosis in gastric cancer patients by remodeling the ECM. With further analysis, we revealed COL1A1, COL5A1, ITGA4, Emilin1, and TSPAN9 as a poor prognostic gene signature for CAF infiltration, with high specificity to stomach adenocarcinoma. This signature

Page $17 / 43$ 
could be translated to the clinic with further studies as a predictive tool for poor prognosis. More importantly, investigating the mechanisms by which the signature genes strengthen the poor prognostic impact of CAFs may put forth new molecular targets for the effective treatment of gastric cancer.

\section{Abbreviations}

ACC: adrenocortical carcinoma

Akt: protein kinase B

AREG: amphiregulin

ASPN: asporin

Bcl-2: B-cell lymphoma 2

BGN: biglycan

BIC: Bioinfo Intelligent Cloud

BLCA: bladder urothelial carcinoma

BP: Gene ontology-biological process

CAF: cancer-associated fibroblast

CC: Gene ontology-cellular compartment

COL1A1: collagen type I alpha 1 chain

COL1A2: collagen type I alpha 2 chain

COL2A1: collagen type II alpha 1 chain

COL3A1: collagen type III alpha 1 chain

COL5A1: collagen type $V$ alpha 1 chain

COL10A1: collagen type $X$ alpha 1 chain

COL11A1: collagen type XI alpha1 chain

CXCL12: C-X-C motif chemokine ligand 12

CYP2C18: cytochrome P450 family 2 subfamily C member 18

CYP2C9: cytochrome P450 family 2 subfamily C member 9 
CYP3A5: cytochrome P450 Family 3 subfamily A member 5

DAVID: The Database for Annotation, Visualization, and Integrated Discovery

DEG: differentially expressed genes

DGIdb: Drug-Gene Interaction database

ECM: extracellular matrix

EFNA5: ephrin A5

EMILIN1: elastin microfibril interface-located protein 1

EMT: epithelial-mesenchymal transition

ERK: extracellular signal-regulated kinase

FAK: focal adhesion kinase

FAP: fibroblast activation protein

FN1: fibronectin 1

GC: Gastric cancer

GEO: Gene Expression Omnibus

GEPIA2: Gene Expression Profiling Interactive Analysis 2

GO: Gene Ontology

GLOBOCAN: The Global Cancer Observatory: CANCER TODAY

GOTERM BP: GO-biological process

GOTERM CC: GO-cellular compartment

GOTERM MF: GO-molecular function

GOTERM: Gene Ontology Term

HER2: Human epidermal growth factor receptor 2

HR: hazard ratio

INHBA: inhibin A 
ITGA4: integrin a4

ITGB1: integrin $\beta 1$

JAM2: junctional adhesion molecule 2

JAM3: junctional adhesion molecule 3

KEGG: Kyoto Encyclopedia of Genes and Genomes

KIRP: kidney renal papillary cell carcinoma

KM plotter: Kaplan-Meier plotter

KM: Kaplan-Meier

LGG: low-grade glioma

Log FC: log2-fold change

LOX: lysyl oxidase

LOXL2: lysyl oxidase-like 2

MADCAM1: mucosal vascular addressin cell adhesion molecule 1

MAPK: mitogen-activated protein kinase

MCODE: Molecular Complex Detection

MCP-Counter: Microenvironment Cell Populations-counter

MESO: Mesothelioma

MF: Gene ontology-molecular function

MMP: matrix metalloproteinase

MMP11: matrix metalloproteinase 11

MT: metallothionein

P53: tumor protein $p 53$

PD-1: programmed cell death protein 1

PI3K: phosphoinositide 3-kinase 
PPI: protein-protein interaction

PTEN: phosphatase and tensin homolog

Ras: rat sarcoma virus

RNA-seq: RNA sequencing

S100A4: S100 calcium-binding protein A4

SERPINH1: serpin family $\mathrm{H}$ member 1

Smad3: SMAD Family Member 3

SPARC: cysteine-rich acidic matrix-associated protein

SPP1: osteopontin (secreted phosphoprotein 1)

STAD: Stomach adenocarcinoma

STRING: The Search Tool for the Retrieval of Interacting Genes

SULF1: sulfatase 1

TCGA: The Cancer Genome Atlas

TGFB: transforming growth factor-beta

TGFB2: transforming growth factor-beta 2

THBS: thrombospondin

THBS1: thrombospondin 1

THBS2: thrombospondin 2

TIDE: Tumor Immune Dysfunction and Exclusion

TIMER2.0: Tumor Immune Estimation Resource 2.0

TIMP1: tissue inhibitor matrix metalloproteinase 1

TME: tumor microenvironment

TSPAN9: tetraspanin 9

UALCAN: University of Alabama Cancer Database 
VCAM-1: vascular cell adhesion protein 1

VCAN: versican

VEGFB: vascular endothelial growth factor B

VEGFR2: vascular endothelial growth factor receptor 2

VLA-4: very late antigen-4

\section{Declarations}

\section{Ethics approval and consent to participate}

Not applicable

Consent for publication

Not applicable

\section{Availability of data and materials}

The GSE13911, GSE29272, GSE79973 and GSE118916 datasets we analyzed during the current study are available in Gene Expression Omnibus (GEO) repository (https://www.ncbi.nlm.nih.gov/geo/geo2r/).

\section{Competing interests}

The authors declare that they have no competing interests.

\section{Funding}

The authors declare that they have not used any funding for the current study.

\section{Authors' contributions}

C.U. analyzed and interpreted the genomic data to identify key hub genes in gastric cancer. G.O. analyzed the prognostic relationship between the key hub genes in gastric cancer and the cancer-associated fibroblasts. Both authors contributed to the writing of the manuscript. G.O. reviewed the manuscript and supervised the whole study. All authors read and approved the final manuscript.

\section{Acknowledgments}

The authors gratefully acknowledge the use of the services and facilities of the Koc University Research Centre for Translational Medicine (KUTTAM) funded by the Presidency of Turkey, Presidency of Strategy and Budget. The content is solely the responsibility of the authors and does not necessarily represent the official views of the Presidency of Strategy and Budget. 


\section{Authors' information}

${ }^{1}$ Graduate School of Health Sciences, Koc University, 34450, Istanbul, Turkey. ${ }^{2}$ Department of Medical Pharmacology, Koc University School of Medicine, 34450, Istanbul, Turkey

\section{References}

1. Sung H, Ferlay J, Siegel RL, Laversanne M, Soerjomataram I, Jemal A, et al. Global Cancer Statistics 2020: GLOBOCAN Estimates of Incidence and Mortality Worldwide for 36 Cancers in 185 Countries. CA: A Cancer Journal for Clinicians. 2021;71(3):209-49.

2. Ajani JA, Lee J, Sano T, Janjigian YY, Fan D, Song S. Gastric adenocarcinoma. Nat Rev Dis Primers. 2017;3:17036.

3. Marin JJG, Perez-Silva L, Macias RIR, Asensio M, Peleteiro-Vigil A, Sanchez-Martin A, et al. Molecular Bases of Mechanisms Accounting for Drug Resistance in Gastric Adenocarcinoma. Cancers (Basel). 2020;12(8).

4. Falzone L, Salomone S, Libra M. Evolution of Cancer Pharmacological Treatments at the Turn of the Third Millennium. Front Pharmacol. 2018;9:1300.

5. Joshi SS, Badgwell BD. Current treatment and recent progress in gastric cancer. CA: A Cancer Journal for Clinicians. 2021;71(3):264-79.

6. Liu T, Zhou L, Li D, Andl T, Zhang Y. Cancer-Associated Fibroblasts Build and Secure the Tumor Microenvironment. Front Cell Dev Biol. 2019;7:60.

7. D'Errico M, de Rinaldis E, Blasi MF, Viti V, Falchetti M, Calcagnile A, et al. Genome-wide expression profile of sporadic gastric cancers with microsatellite instability. Eur J Cancer. 2009;45(3):461-9.

8. Li W-Q, Hu N, Burton VH, Yang HH, Su H, Conway CM, et al. PLCE1 mRNA and Protein Expression and Survival of Patients with Esophageal Squamous Cell Carcinoma and Gastric Adenocarcinoma. Cancer Epidemiology Biomarkers \&amp; Prevention. 2014;23(8):1579-88.

9. He J, Jin Y, Chen Y, Yao HB, Xia YJ, Ma YY, et al. Downregulation of ALDOB is associated with poor prognosis of patients with gastric cancer. Onco Targets Ther. 2016;9:6099-109.

10. Li L, Zhu Z, Zhao Y, Zhang Q, Wu X, Miao B, et al. FN1, SPARC, and SERPINE1 are highly expressed and significantly related to a poor prognosis of gastric adenocarcinoma revealed by microarray and bioinformatics. Sci Rep. 2019;9(1):7827.

11. Huang da W, Sherman BT, Lempicki RA. Systematic and integrative analysis of large gene lists using DAVID bioinformatics resources. Nat Protoc. 2009;4(1):44-57.

12. Zhou Y, Zhou B, Pache L, Chang M, Khodabakhshi AH, Tanaseichuk O, et al. Metascape provides a biologist-oriented resource for the analysis of systems-level datasets. Nat Commun. 2019;10(1):1523.

13. Szklarczyk D, Gable AL, Lyon D, Junge A, Wyder S, Huerta-Cepas J, et al. STRING v11: protein-protein association networks with increased coverage, supporting functional discovery in genome-wide 
experimental datasets. Nucleic Acids Res. 2019;47(D1):D607-D13.

14. Shannon P, Markiel A, Ozier O, Baliga NS, Wang JT, Ramage D, et al. Cytoscape: a software environment for integrated models of biomolecular interaction networks. Genome Res. 2003;13(11):2498-504.

15. Bader GD, Hogue CWV. An automated method for finding molecular complexes in large protein interaction networks. BMC Bioinformatics. 2003;4(1):2.

16. Chin $\mathrm{CH}$, Chen $\mathrm{SH}$, Wu HH, Ho CW, Ko MT, Lin CY. cytoHubba: identifying hub objects and subnetworks from complex interactome. BMC Syst Biol. 2014;8 Suppl 4(Suppl 4):S11.

17. Chandrashekar DS, Bashel B, Balasubramanya SAH, Creighton CJ, Ponce-Rodriguez I, Chakravarthi B, et al. UALCAN: A Portal for Facilitating Tumor Subgroup Gene Expression and Survival Analyses. Neoplasia. 2017;19(8):649-58.

18. Tang Z, Kang B, Li C, Chen T, Zhang Z. GEPIA2: an enhanced web server for large-scale expression profiling and interactive analysis. Nucleic Acids Res. 2019;47(W1):W556-W60.

19. Li T, Fu J, Zeng Z, Cohen D, Li J, Chen Q, et al. TIMER2.0 for analysis of tumor-infiltrating immune cells. Nucleic Acids Res. 2020;48(W1):W509-w14.

20. Freshour SL, Kiwala S, Cotto KC, Coffman AC, McMichael JF, Song JJ, et al. Integration of the DrugGene Interaction Database (DGIdb 4.0) with open crowdsource efforts. Nucleic Acids Res. 2021;49(D1):D1144-d51.

21. Henkel G, Krebs B. Metallothioneins: zinc, cadmium, mercury, and copper thiolates and selenolates mimicking protein active site features--structural aspects and biological implications. Chem Rev. 2004;104(2):801-24.

22. Gelse K, Pöschl E, Aigner T. Collagens-structure, function, and biosynthesis. Adv Drug Deliv Rev. 2003;55(12):1531-46.

23. Nissen NI, Karsdal M, Willumsen N. Collagens and Cancer associated fibroblasts in the reactive stroma and its relation to cancer biology. Journal of Experimental \& Clinical Cancer Research. 2019;38(1):115.

24. Huang J, Zhang L, Wan D, Zhou L, Zheng S, Lin S, et al. Extracellular matrix and its therapeutic potential for cancer treatment. Signal Transduction and Targeted Therapy. 2021;6(1):153.

25. Podhajcer OL, Benedetti L, Girotti MR, Prada F, Salvatierra E, Llera AS. The role of the matricellular protein SPARC in the dynamic interaction between the tumor and the host. Cancer Metastasis Rev. 2008;27(3):523-37.

26. Liu B, Chen X, Zhan Y, Wu B, Pan S. Identification of a Gene Signature for Renal Cell CarcinomaAssociated Fibroblasts Mediating Cancer Progression and Affecting Prognosis. Frontiers in Cell and Developmental Biology. 2021;8(1914).

27. Grunberg N, Pevsner-Fischer M, Goshen-Lago T, Diment J, Stein Y, Lavon H, et al. Cancer-Associated Fibroblasts Promote Aggressive Gastric Cancer Phenotypes via Heat Shock Factor 1-Mediated Secretion of Extracellular Vesicles. Cancer Res. 2021;81(7):1639-53. 
28. Qin Y, Wang F, Ni H, Liu Y, Yin Y, Zhou X, et al. Cancer-associated fibroblasts in gastric cancer affect malignant progression via the CXCL12-CXCR4 axis. J Cancer. 2021;12(10):3011-23.

29. Liu X, Yao L, Qu J, Liu L, Lu N, Wang J, et al. Cancer-associated fibroblast infiltration in gastric cancer: the discrepancy in subtypes pathways and immunosuppression. Journal of Translational Medicine. 2021;19(1):325.

30. Izumi D, Ishimoto T, Miyake K, Sugihara H, Eto K, Sawayama H, et al. CXCL12/CXCR4 activation by cancer-associated fibroblasts promotes integrin $\beta 1$ clustering and invasiveness in gastric cancer. Int J Cancer. 2016;138(5):1207-19.

31. Ma Y, Zhu J, Chen S, Li T, Ma J, Guo S, et al. Activated gastric cancer-associated fibroblasts contribute to the malignant phenotype and 5-FU resistance via paracrine action in gastric cancer. Cancer Cell Int. 2018;18:104.

32. Su C-Y, Li J-Q, Zhang L-L, Wang H, Wang F-H, Tao Y-W, et al. The Biological Functions and Clinical Applications of Integrins in Cancers. Frontiers in pharmacology. 2020;11:579068-

33. Schlesinger M, Bendas G. Contribution of very late antigen-4 (VLA-4) integrin to cancer progression and metastasis. Cancer Metastasis Rev. 2015;34(4):575-91.

34. Postigo AA, Sánchez-Mateos P, Lazarovits Al, Sánchez-Madrid F, de Landázuri MO. Alpha 4 beta 7 integrin mediates $B$ cell binding to fibronectin and vascular cell adhesion molecule-1. Expression and function of alpha 4 integrins on human B lymphocytes. The Journal of Immunology. 1993;151(5):2471-83.

35. Sechler JL, Cumiskey AM, Gazzola DM, Schwarzbauer JE. A novel RGD-independent fibronectin assembly pathway initiated by alpha4beta 1 integrin binding to the alternatively spliced $\mathrm{V}$ region. $\mathrm{J}$ Cell Sci. 2000;113 (Pt 8):1491-8.

36. Zhu N, Eves PC, Katerinaki E, Szabo M, Morandini R, Ghanem G, et al. Melanoma cell attachment, invasion, and integrin expression is upregulated by tumor necrosis factor alpha and suppressed by alpha melanocyte stimulating hormone. J Invest Dermatol. 2002;119(5):1165-71.

37. Liu CC, Leclair P, Yap SQ, Lim CJ. The membrane-proximal KXGFFKR motif of a-integrin mediates chemoresistance. Mol Cell Biol. 2013;33(21):4334-45.

38. Calzada MJ, Zhou L, Sipes JM, Zhang J, Krutzsch HC, Iruela-Arispe ML, et al. Alpha4beta1 integrin mediates selective endothelial cell responses to thrombospondins 1 and 2 in vitro and modulates angiogenesis in vivo. Circ Res. 2004;94(4):462-70.

39. Bayless KJ, Davis GE. Identification of dual alpha 4beta1 integrin binding sites within a 38 amino acid domain in the $\mathrm{N}$-terminal thrombin fragment of human osteopontin. $\mathrm{J}$ Biol Chem. 2001;276(16):13483-9.

40. Maiorani O, Pivetta E, Capuano A, Modica TME, Wassermann B, Bucciotti F, et al. Neutrophil elastase cleavage of the gC1q domain impairs the EMILIN1-a4ß1 integrin interaction, cell adhesion and antiproliferative activity. Scientific Reports. 2017;7(1):39974.

41. Baiula M, Spampinato $S$, Gentilucci L, Tolomelli A. Novel Ligands Targeting $a(4) \beta(1)$ Integrin: Therapeutic Applications and Perspectives. Front Chem. 2019;7:489. 
42. Danussi C, Petrucco A, Wassermann B, Pivetta E, Modica TME, Belluz LDB, et al. EMILIN1-a4/a9 integrin interaction inhibits dermal fibroblast and keratinocyte proliferation. Journal of Cell Biology. 2011;195(1):131-45.

43. Spessotto P, Cervi M, Mucignat MT, Mungiguerra G, Sartoretto I, Doliana R, et al. beta 1 Integrindependent cell adhesion to EMILIN-1 is mediated by the gC1q domain. J Biol Chem. 2003;278(8):6160-7.

44. Danussi C, Petrucco A, Wassermann B, Modica TM, Pivetta E, Del Bel Belluz L, et al. An EMILIN1negative microenvironment promotes tumor cell proliferation and lymph node invasion. Cancer Prev Res (Phila). 2012;5(9):1131-43.

45. Salani R, Neuberger I, Kurman RJ, Bristow RE, Chang HW, Wang TL, et al. Expression of extracellular matrix proteins in ovarian serous tumors. Int J Gynecol Pathol. 2007;26(2):141-6.

46. Rao UN, Hood BL, Jones-Laughner JM, Sun M, Conrads TP. Distinct profiles of oxidative stressrelated and matrix proteins in adult bone and soft tissue osteosarcoma and desmoid tumors: a proteomics study. Hum Pathol. 2013;44(5):725-33.

47. Qi Y, Lv J, Liu S, Sun L, Wang Y, Li H, et al. TSPAN9 and EMILIN1 synergistically inhibit the migration and invasion of gastric cancer cells by increasing TSPAN9 expression. BMC Cancer. 2019;19(1):630.

48. Li PY, Lv J, Qi WW, Zhao SF, Sun LB, Liu N, et al. Tspan9 inhibits the proliferation, migration, and invasion of human gastric cancer SGC7901 cells via the ERK1/2 pathway. Oncol Rep. 2016;36(1):448-54.

49. Deng Y, Cai S, Shen J, Peng H. Tetraspanins: Novel Molecular Regulators of Gastric Cancer. Front Oncol. 2021;11:702510.

50. Shima H, Inagaki A, Imura T, Yamagata Y, Watanabe K, Igarashi K, et al. Collagen V Is a Potential Substrate for Clostridial Collagenase $\mathrm{G}$ in Pancreatic Islet Isolation. Journal of Diabetes Research. 2016;2016:4396756.

51. Syed YY, Dhillon S. Ocriplasmin: a review of its use in patients with symptomatic vitreomacular adhesion. Drugs. 2013;73(14):1617-25.

52. Carter MJ, Gilligan AM, Waycaster CR, Fife CE. Treating pressure ulcers with clostridial collagenase ointment: Results from the US Wound Registry. Wound Repair Regen. 2016;24(5):904-12.

53. Warwick D, Arandes-Renú JM, Pajardi G, Witthaut J, Hurst LC. Collagenase Clostridium histolyticum: emerging practice patterns and treatment advances. Journal of Plastic Surgery and Hand Surgery. 2016;50(5):251-61.

54. Dolor A, Szoka FC, Jr. Digesting a Path Forward: The Utility of Collagenase Tumor Treatment for Improved Drug Delivery. Mol Pharm. 2018;15(6):2069-83.

55. García-Olmo D, Villarejo Campos P, Barambio J, Gomez-Heras SG, Vega-Clemente L, OlmedillasLopez S, et al. Intraperitoneal collagenase as a novel therapeutic approach in an experimental model of colorectal peritoneal carcinomatosis. Scientific Reports. 2021;11(1):503.

56. Qu T, Li YP, Li XH, Chen Y. Identification of potential biomarkers and drugs for papillary thyroid cancer based on gene expression profile analysis. Mol Med Rep. 2016;14(6):5041-8. 
57. Pines M. Halofuginone for fibrosis, regeneration and cancer in the gastrointestinal tract. World $\mathrm{J}$ Gastroenterol. 2014;20(40):14778-86.

58. Pines M, Spector I. Halofuginone - the multifaceted molecule. Molecules. 2015;20(1):573-94.

59. Sheffer Y, Leon O, Pinthus JH, Nagler A, Mor Y, Genin O, et al. Inhibition of fibroblast to myofibroblast transition by halofuginone contributes to the chemotherapy-mediated antitumoral effect. Mol Cancer Ther. 2007;6(2):570-7.

60. Spector I, Zilberstein Y, Lavy A, Nagler A, Genin O, Pines M. Involvement of host stroma cells and tissue fibrosis in pancreatic tumor development in transgenic mice. PLoS One. 2012;7(7):e41833-e.

61. Sandborn WJ, Cyrille M, Hansen MB, Feagan BG, Loftus EV, Jr., Rogler G, et al. Efficacy and Safety of Abrilumab in a Randomized, Placebo-Controlled Trial for Moderate-to-Severe Ulcerative Colitis. Gastroenterology. 2019;156(4):946-57.e18.

62. Luzentales-Simpson M, Pang YCF, Zhang A, Sousa JA, Sly LM. Vedolizumab: Potential Mechanisms of Action for Reducing Pathological Inflammation in Inflammatory Bowel Diseases. Front Cell Dev Biol. 2021;9:612830.

63. Miller DH, Weber T, Grove R, Wardell C, Horrigan J, Graff O, et al. Firategrast for relapsing remitting multiple sclerosis: a phase 2, randomised, double-blind, placebo-controlled trial. Lancet Neurol. 2012;11(2):131-9.

64. Abraham WM, Gill A, Ahmed A, Sielczak MW, Lauredo IT, Botinnikova Y, et al. A small-molecule, tightbinding inhibitor of the integrin alpha(4)beta(1) blocks antigen-induced airway responses and inflammation in experimental asthma in sheep. Am J Respir Crit Care Med. 2000;162(2 Pt 1):603-11.

65. Winkler J, Abisoye-Ogunniyan A, Metcalf $\mathrm{KJ}$, Werb Z. Concepts of extracellular matrix remodelling in tumour progression and metastasis. Nature Communications. 2020;11(1):5120.

66. Henke E, Nandigama R, Ergün S. Extracellular Matrix in the Tumor Microenvironment and Its Impact on Cancer Therapy. Front Mol Biosci. 2020;6:160-

67. Petersen EV, Chudakova DA, Skorova EY, Anikin V, Reshetov IV, Mynbaev OA. The Extracellular MatrixDerived Biomarkers for Diagnosis, Prognosis, and Personalized Therapy of Malignant Tumors. Front Oncol. 2020;10:575569.

68. Moreira AM, Pereira J, Melo S, Fernandes MS, Carneiro P, Seruca R, et al. The Extracellular Matrix: An Accomplice in Gastric Cancer Development and Progression. Cells. 2020;9(2):394.

69. Zhao Y, Zhou T, Li A, Yao H, He F, Wang L, et al. A Potential Role of Collagens Expression in Distinguishing Between Premalignant and Malignant Lesions in Stomach. The Anatomical Record. 2009;292(5):692-700.

70. Li J, Ding Y, Li A. Identification of COL1A1 and COL1A2 as candidate prognostic factors in gastric cancer. World J Surg Oncol. 2016;14(1):297-.

71. Wang J, Gao P, Song Y, Sun J, Chen X, Yu H, et al. Prognostic value of gastric cancer-associated gene signatures: Evidence based on a meta-analysis using integrated bioinformatics methods. J Cell Mol Med. 2018;22(11):5743-7. 
72. Li Y, Wang J-S, Zhang T, Wang H-C, Li L-P. Identification of New Therapeutic Targets for Gastric Cancer With Bioinformatics. Frontiers in Genetics. 2020;11(865).

73. Zeng D, Li M, Zhou R, Zhang J, Sun H, Shi M, et al. Tumor Microenvironment Characterization in Gastric Cancer Identifies Prognostic and Immunotherapeutically Relevant Gene Signatures. Cancer Immunol Res. 2019;7(5):737-50.

74. Zhou ZH, Ji CD, Xiao HL, Zhao HB, Cui YH, Bian XW. Reorganized Collagen in the Tumor Microenvironment of Gastric Cancer and Its Association with Prognosis. J Cancer. 2017;8(8):146676.

75. Capuano A, Pivetta E, Sartori G, Bosisio G, Favero A, Cover E, et al. Abrogation of EMILIN1- $\beta 1$ integrin interaction promotes experimental colitis and colon carcinogenesis. Matrix Biol. 2019;83:97-115.

76. Zhao Y, Zhang X, Yao J, Jin Z, Liu C. Expression patterns and the prognostic value of the EMILIN/Multimerin family members in low-grade glioma. PeerJ. 2020;8:e8696.

77. Qi Y, Qi W, Liu S, Sun L, Ding A, Yu G, et al. TSPAN9 suppresses the chemosensitivity of gastric cancer to 5-fluorouracil by promoting autophagy. Cancer Cell Int. 2020;20:4.

78. Feng T, Sun L, Qi W, Pan F, Lv J, Guo J, et al. Prognostic significance of Tspan9 in gastric cancer. Mol Clin Oncol. 2016;5(3):231-6.

79. Pivetta E. A Rare Bird among Major Extracellular Matrix Proteins: EMILIN1 and the Tumor Suppressor Function. Journal of Carcinogenesis \& Mutagenesis. 2013.

80. Amor López A, Mazariegos MS, Capuano A, Ximénez-Embún P, Hergueta-Redondo M, Recio JÁ, et al. Inactivation of EMILIN-1 by Proteolysis and Secretion in Small Extracellular Vesicles Favors Melanoma Progression and Metastasis. International Journal of Molecular Sciences. 2021;22(14).

81. Bonnans C, Chou J, Werb Z. Remodelling the extracellular matrix in development and disease. Nat Rev Mol Cell Biol. 2014;15(12):786-801.

82. Racle J, de Jonge K, Baumgaertner P, Speiser DE, Gfeller D. Simultaneous enumeration of cancer and immune cell types from bulk tumor gene expression data. Elife. 2017;6.

83. Becht E, Giraldo NA, Lacroix L, Buttard B, Elarouci N, Petitprez F, et al. Estimating the population abundance of tissue-infiltrating immune and stromal cell populations using gene expression. Genome Biol. 2016;17(1):218.

84. Aran D, Hu Z, Butte AJ. xCell: digitally portraying the tissue cellular heterogeneity landscape. Genome Biol. 2017;18(1):220.

85. Jiang P, Gu S, Pan D, Fu J, Sahu A, Hu X, et al. Signatures of T cell dysfunction and exclusion predict cancer immunotherapy response. Nat Med. 2018;24(10):1550-8.

86. Alping P, Askling J, Burman J, Fink K, Fogdell-Hahn A, Gunnarsson M, et al. Cancer Risk for Fingolimod, Natalizumab, and Rituximab in Multiple Sclerosis Patients. Ann Neurol. 2020;87(5):68899.

\section{Tables}


Table 1 Functional annotation clustering of 83 differentially expressed genes in gastric cancer. Analysis was performed with the highest classification stringency in DAVID (ECM: Extracellular Matrix, GOTERM: Gene Ontology Term, GOTERM BP: GO-biological process, GOTERM MF: GO-molecular function and GOTERM CC: GO-cellular compartment, KEGG Pathway: Pathways listed in Kyoto Encyclopedia of Genes and Genomes).

\begin{tabular}{|c|c|c|c|c|c|}
\hline $\begin{array}{l}\text { Annotation } \\
\text { Cluster } 1\end{array}$ & Enrichment Score: 7.26 & Count & Genes & $\begin{array}{l}\text { P- } \\
\text { Value }\end{array}$ & Benjamini \\
\hline $\begin{array}{l}\text { KEGG } \\
\text { Pathway }\end{array}$ & ECM-receptor interaction & 12 & \multirow{3}{*}{$\begin{array}{l}\text { COL1A1, COL 1A2, } \\
\text { COL2A1, COL 3A1, } \\
\text { COL4A1, COL5A1, } \\
\text { COL5A2, COL6A3, FN1, } \\
\text { SPP1, THBS1, THBS2 }\end{array}$} & $\begin{array}{l}2.2 \mathrm{E}- \\
11\end{array}$ & $1.6 \mathrm{E}-9$ \\
\hline $\begin{array}{l}\text { KEGG } \\
\text { Pathway }\end{array}$ & Focal adhesion & 12 & & $2.2 . \mathrm{E}-$ & $5.2 \mathrm{E}-6$ \\
\hline $\begin{array}{l}\text { KEGG } \\
\text { Pathway }\end{array}$ & PI3K-Akt signaling pathway & 12 & & $\begin{array}{l}3.4 \mathrm{E}- \\
5\end{array}$ & $4.0 \mathrm{E}-4$ \\
\hline $\begin{array}{l}\text { Annotation } \\
\text { Cluster } 2\end{array}$ & Enrichment Score: 6.68 & Count & & $\begin{array}{l}\text { P- } \\
\text { Value }\end{array}$ & Benjamini \\
\hline $\begin{array}{l}\text { GOTERM } \\
\text { BP DIRECT }\end{array}$ & $\begin{array}{l}\text { Negative regulation of } \\
\text { growth }\end{array}$ & 6 & \multirow{3}{*}{$\begin{array}{l}\text { MT1E, MT1F, MT1G, } \\
\text { MT1H, MT1M, MT1X }\end{array}$} & $\begin{array}{l}2.4 \mathrm{E}- \\
8\end{array}$ & $3.1 \mathrm{E}-6$ \\
\hline $\begin{array}{l}\text { GOTERM } \\
\text { BP DIRECT }\end{array}$ & Cellular response to zinc ion & 6 & & $\begin{array}{l}2.4 \mathrm{E}- \\
8\end{array}$ & $3.1 \mathrm{E}-6$ \\
\hline $\begin{array}{l}\text { KEGG } \\
\text { Pathway }\end{array}$ & Mineral absorption & 6 & & $\begin{array}{l}1.6 \mathrm{E}- \\
5\end{array}$ & $2.3 \mathrm{E}-4$ \\
\hline $\begin{array}{l}\text { Annotation } \\
\text { Cluster } 3\end{array}$ & Enrichment Score: 1.25 & Count & & $\begin{array}{l}\text { P- } \\
\text { Value }\end{array}$ & Benjamini \\
\hline $\begin{array}{l}\text { GOTERM } \\
\text { MF DIRECT }\end{array}$ & Oxygen binding & 3 & \multirow[t]{7}{*}{$\begin{array}{l}\text { CYP2C18, CYP2C9, } \\
\text { CYP } 3 A 5\end{array}$} & $2.0 \mathrm{E}-$ & $2.4 \mathrm{E}-1$ \\
\hline $\begin{array}{l}\text { GOTERM } \\
\text { MF DIRECT }\end{array}$ & $\begin{array}{l}\text { Oxidoreductase activity, } \\
\text { acting on paired donors } \\
\text { with incorporation or } \\
\text { reduction of molecular } \\
\text { oxygen }\end{array}$ & 3 & & $2.9 \mathrm{E}-$ & $2.9 \mathrm{E}-1$ \\
\hline $\begin{array}{l}\text { GOTERM } \\
\text { MF DIRECT }\end{array}$ & Monooxygenase activity & 3 & & $2.9 \mathrm{E}-$ & $2.9 \mathrm{E}-1$ \\
\hline $\begin{array}{l}\text { GOTERM } \\
\text { CC DIRECT }\end{array}$ & Organelle membrane & 3 & & $\begin{array}{l}5.9 \mathrm{E}- \\
2\end{array}$ & $3.2 \mathrm{E}-1$ \\
\hline $\begin{array}{l}\text { KEGG } \\
\text { Pathway }\end{array}$ & Retinol metabolism & 3 & & $\begin{array}{l}8.1 \mathrm{E}- \\
2\end{array}$ & $4.8 \mathrm{E}-1$ \\
\hline $\begin{array}{l}\text { GOTERM } \\
\text { MF DIRECT }\end{array}$ & Heme binding & 3 & & $\begin{array}{l}1.3 \mathrm{E}- \\
1\end{array}$ & 6.7E-1 \\
\hline $\begin{array}{l}\text { GOTERM } \\
\text { MF DIRECT }\end{array}$ & Iron ion binding & 3 & & $l_{1}^{1.6 \mathrm{E}-}$ & $7.8 \mathrm{E}-1$ \\
\hline
\end{tabular}


Table 2 Topological parameters for six upregulated extracellular matrix hub genes with the highest degree.

\begin{tabular}{lllllll}
$\begin{array}{l}\text { Gene } \\
\text { Symbol }\end{array}$ & Gene & Degree & $\begin{array}{l}\text { Closeness } \\
\text { of } \\
\text { Centrality }\end{array}$ & $\begin{array}{l}\text { Clustering } \\
\text { Coefficient }\end{array}$ & $\begin{array}{l}\text { Average } \\
\text { Shortest } \\
\text { Path Length }\end{array}$ & $\begin{array}{l}\text { Neighborhood } \\
\text { Connectivity }\end{array}$ \\
\hline COL3A1 & $\begin{array}{l}\text { collagen type III } \\
\text { alpha 1 chain }\end{array}$ & 16 & 0.71 & 0.52 & 1.41 & 10.12 \\
\hline FN1 & $\begin{array}{l}\text { fibronectin 1 } \\
\text { COL1A2 }\end{array}$ & 16 & 0.69 & 0.43 & 1.44 & 9.68 \\
\hline $\begin{array}{l}\text { collagen type I } \\
\text { alpha 2 chain }\end{array}$ & 15 & 0.68 & 0.59 & 1.48 & 10.80 \\
\hline COL1A1 & $\begin{array}{l}\text { collagen type I } \\
\text { alpha 1 chain }\end{array}$ & 15 & 0.68 & 0.62 & 1.48 & 11.26 \\
\hline COL5A1 & $\begin{array}{l}\text { collagen type V } \\
\text { alpha 1 chain }\end{array}$ & 13 & 0.64 & 0.76 & 1.56 & 12.15 \\
\hline SPARC & $\begin{array}{l}\text { secreted protein } \\
\text { acidic and } \\
\text { cysteine rich }\end{array}$ & 13 & 0.64 & 0.58 & 1.56 & 11.38 \\
\hline
\end{tabular}

Table 3 Parameters of the multivariate Cox proportional regression model for six key genes and cancerassociated fibroblasts. TCGA data for stomach adenocarcinoma samples were analyzed in the TIMER2.0 immune association - gene outcome module using the TIDE algorithm for the allocation of samples to the high vs. Iow CAF infiltration groups. Likelihood ratio and Score log-rank tests were performed (CAF: cancer-associated fibroblast, Cl: confidence interval, HR: hazard ratio). 


\begin{tabular}{|c|c|c|c|c|}
\hline Variate/s & $\begin{array}{l}\text { HR of CAF } \\
\text { infiltration }\end{array}$ & $95 \% \mathrm{Cl}$ & $\begin{array}{l}\text { z- } \\
\text { score }\end{array}$ & p-value \\
\hline CAF & 5.240 & $\begin{array}{l}2.004- \\
13.702\end{array}$ & 3.377 & 0.001 \\
\hline $\mathrm{CAF}, \mathrm{COL1A1}$ & 5.430 & $\begin{array}{l}1.392- \\
21.179\end{array}$ & 2.437 & 0.015 \\
\hline CAF, COL1A2 & 5.631 & $\begin{array}{l}1.218- \\
26.033\end{array}$ & 2.212 & 0.027 \\
\hline CAF, COL3A1 & 5.916 & $\begin{array}{l}1.078- \\
32.465\end{array}$ & 2.047 & 0.041 \\
\hline CAF, COL5A1 & 8.584 & $\begin{array}{l}1.767- \\
41.695\end{array}$ & 2.666 & 0.008 \\
\hline CAF, FN1 & 4.247 & $\begin{array}{l}0.956- \\
18.880\end{array}$ & 1.900 & 0.057 \\
\hline CAF, SPARC & 3.042 & $0.599-0.878$ & 1.342 & 0.180 \\
\hline $\mathrm{CAF}, \mathrm{COL1A1}, \mathrm{COL1A2}$ & 5.789 & $\begin{array}{l}1.164- \\
28.802\end{array}$ & 2.145 & 0.032 \\
\hline CAF, COL1A1, COL3A1 & 6.167 & $\begin{array}{l}1.034- \\
36.775\end{array}$ & 1.997 & 0.046 \\
\hline CAF, COL1A1, COL5A1 & 11.654 & $\begin{array}{l}2.247- \\
60.438\end{array}$ & 2.924 & 0.003 \\
\hline CAF, COL1A2, COL3A1 & 5.968 & $\begin{array}{l}1.074- \\
33.168\end{array}$ & 2.041 & 0.041 \\
\hline CAF, COL1A2, COL5A1 & 7.701 & $\begin{array}{l}1.550- \\
38.253\end{array}$ & 2.496 & 0.013 \\
\hline CAF, COL3A1, COL5A1 & 6.339 & $\begin{array}{l}1.151- \\
34.913\end{array}$ & 2.121 & 0.034 \\
\hline $\begin{array}{l}\text { CAF, COL1A1, COL1A2, COL3A1, } \\
\text { COL } 5 A 1\end{array}$ & 10.176 & $\begin{array}{l}1.532- \\
67.610\end{array}$ & 2.401 & 0.016 \\
\hline
\end{tabular}

Table 4 Parameters of the stepwise multivariate Cox proportional regression model for cancer-associated fibroblasts and signature genes. TCGA data for stomach adenocarcinoma samples were analyzed in the TIMER2.0 immune association - gene outcome module using the TIDE algorithm for the allocation of samples to the high vs. low CAF infiltration groups. Likelihood ratio and Score log-rank tests were performed (CAF: cancer-associated fibroblast, Cl: confidence interval, HR: hazard ratio). 


\section{Variate/s}

\begin{tabular}{l} 
CAF, COL1A1, COL5A1 \\
CAF, COL1A1, COL5A1, ITGA4 \\
CAF, COL1A1, COL5A1, ITGA4, EMILIN1 \\
CAF, COL1A1, COL5A1, ITGA4, EMILIN1, \\
TSPAN9 \\
\hline
\end{tabular}

HR of CAF

infiltration
$95 \% \mathrm{Cl}$

\section{Adrenocortical Carcinoma}

CAF, COL1A1, COL5A1

0.011

0.011

0.007

0.014 CAF, COL
TSPAN9

CAF, COL1A1, COL5A1, ITGA4

CAF, COL1A1, COL5A1, ITGA4, EMILIN1

CAF, COL1A1, COL5A1, ITGA4, EMILIN1,
11.654

12.247

28.315

36.813

\section{Stomach Adenocarcinoma}

$$
60.438
$$

$\begin{array}{lll}2.335- & 2.963 & 0.003 \\ 64.240 & & \end{array}$

$\begin{array}{lll}4.143- & 3.409 & 0.001 \\ 193.524 & & \end{array}$

$\begin{array}{lll}5.007- & 3.543 & 0.000 \\ 270.645 & & \end{array}$

$\begin{array}{ll}\mathrm{z}- & \mathrm{p}- \\ \text { score } & \text { value }\end{array}$

score value




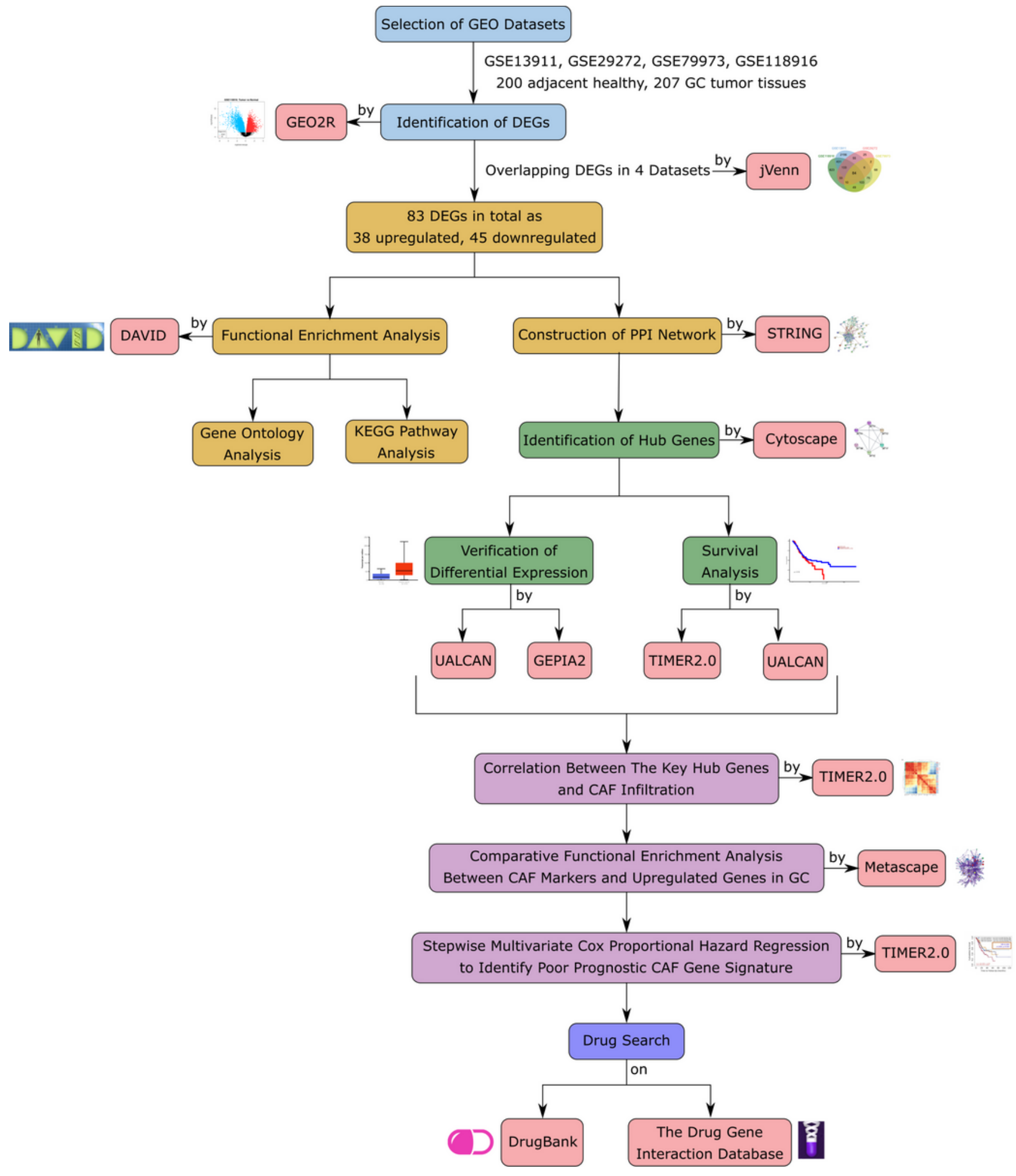

\section{Figure 1}

The workflow in the study. GSE13911, GSE29272, GSE79973, GSE118916 denote four expression profiling datasets from the GEO database. CAF: cancer-associated fibroblast, DAVID: The Database for Annotation, Visualization, and Integrated Discovery, DEG: differentially expressed genes, GEPIA2: Gene Expression Profiling Interactive Analysis 2, PPI: protein-protein interaction, UALCAN: University of Alabama Cancer Database. 
A
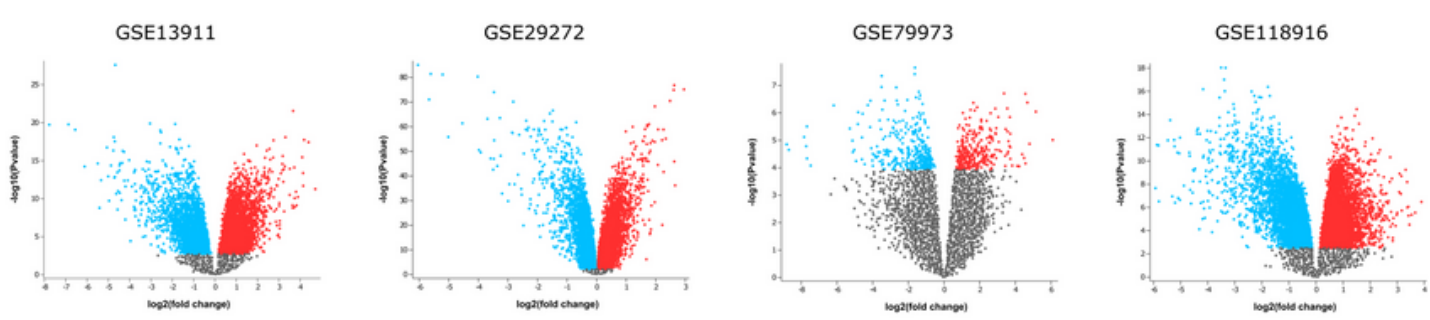

- Upregulated gene Downregulated gene

\begin{tabular}{|c|c|c|c|c|}
\hline \# of genes\Dataset & GSE13911 & GSE29272 & GSE79973 & GSE118916 \\
\hline Upregulated & 943 & 175 & 144 & 848 \\
\hline Downregulated & 2115 & 173 & 252 & 818 \\
\hline Total DEGs & 3058 & 348 & 396 & 1666 \\
\hline
\end{tabular}

B

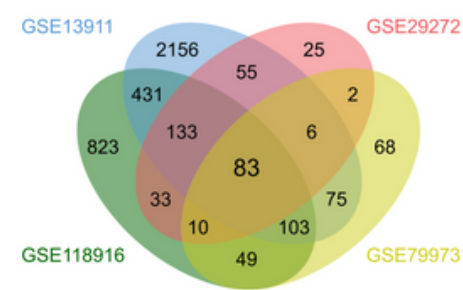

C Upregulated genes

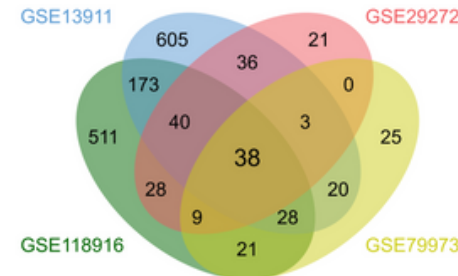

D Downregulated genes

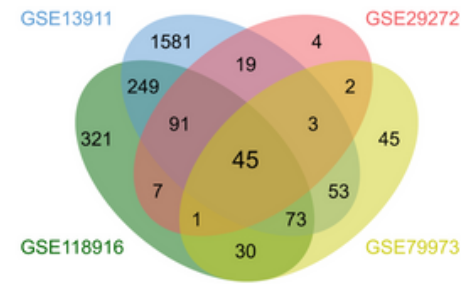

Most Enriched GO Terms for Downregulated Genes

$\mathbf{E}$

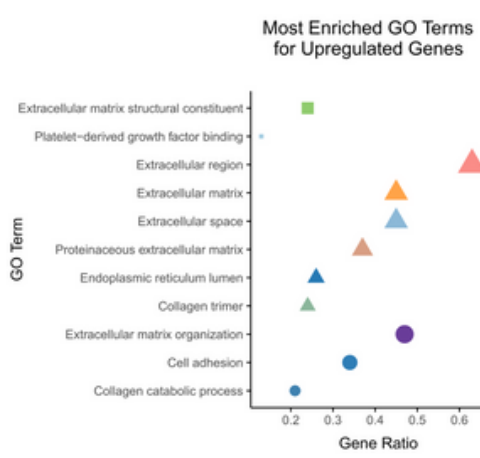

$\mathbf{F}$

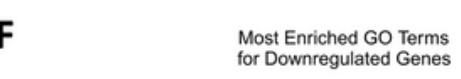

G
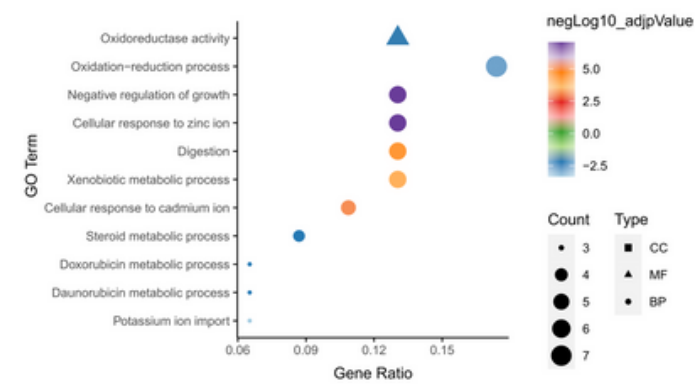

Most Enriched KEGG Pathways

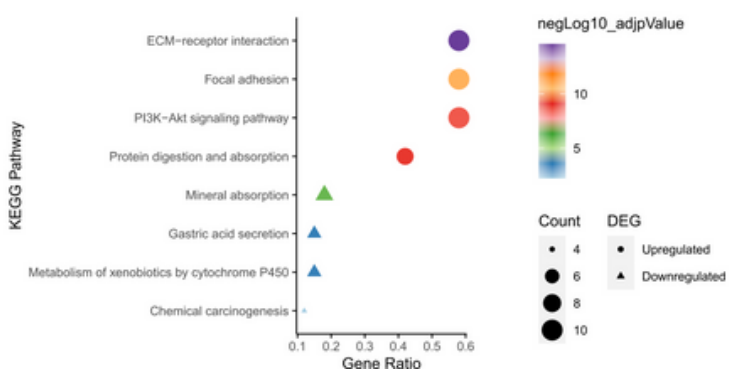

Figure 2

Identification and functional enrichment analysis of differentially expressed genes in gastric cancer. A Volcano plots of differentially expressed genes (DEGs) in four GEO datasets with the number of upregulated, downregulated genes and, the total number of DEGs detected at each dataset. Overlapping $\mathbf{B}$ DEGs, $\mathbf{C}$ upregulated genes and $\mathbf{D}$ downregulated genes in four GEO datasets. Bubble plots of the most 
enriched $\mathrm{GO}$ terms for $\mathbf{E}$ upregulated genes, $\mathrm{F}$ downregulated genes, and $\mathbf{G}$ KEGG pathways for all DEGs (BP: GO-biological process, MF: GO-molecular function, and CC: GO-cellular compartment).

A

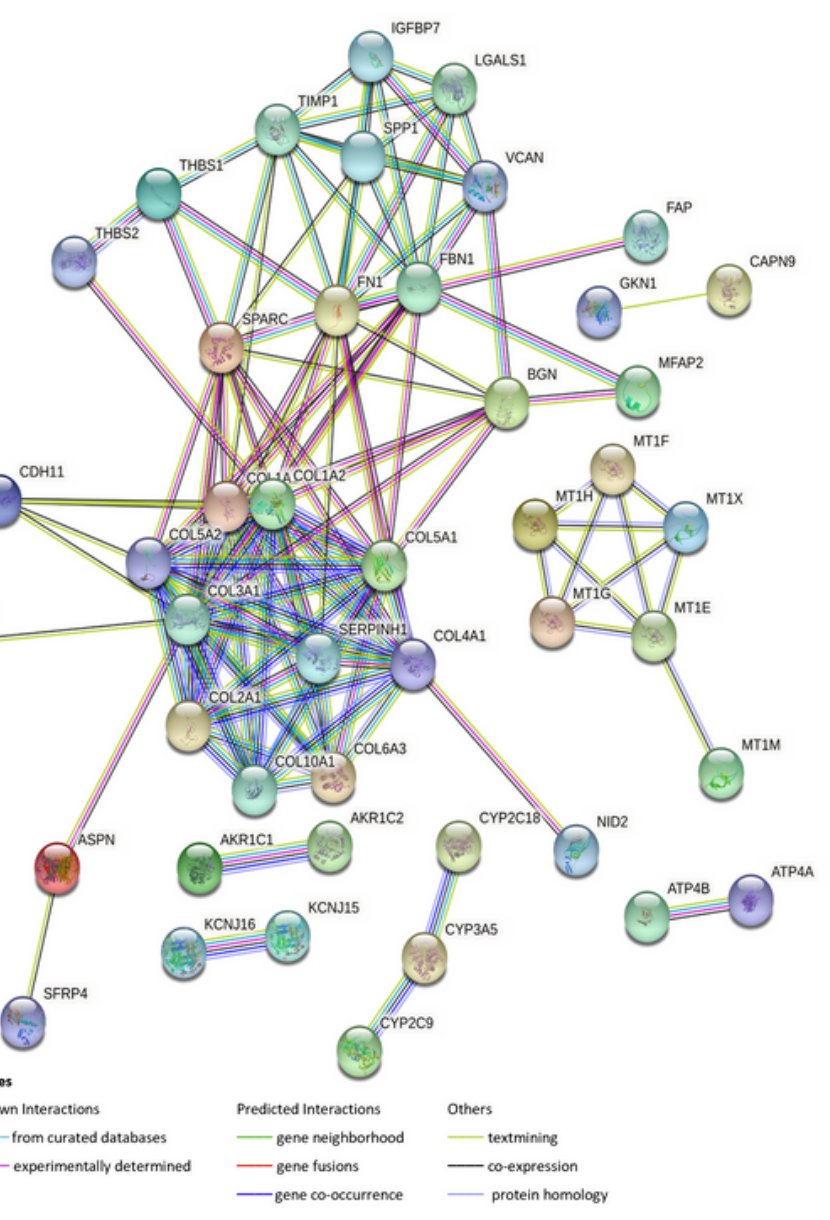

C Module 1

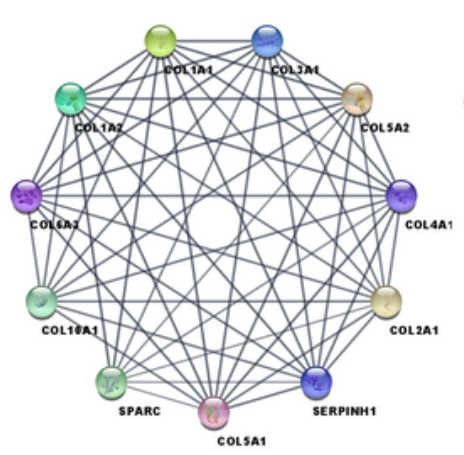

Module 2
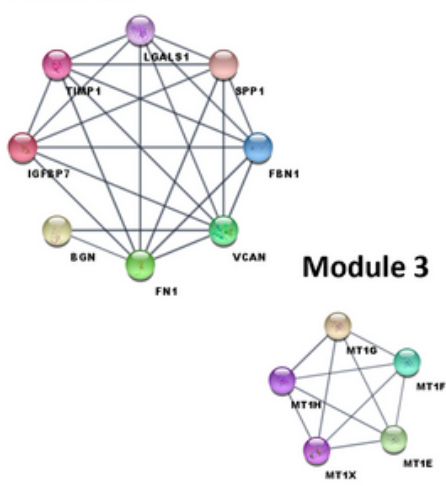

\begin{tabular}{|cccc|}
\hline Module & Score & \#Nodes & \#Edges \\
\hline 1 & 10.6 & 11 & 53 \\
\hline 2 & 6.6 & 8 & 23 \\
\hline 3 & 5 & 5 & 10 \\
\hline
\end{tabular}

B
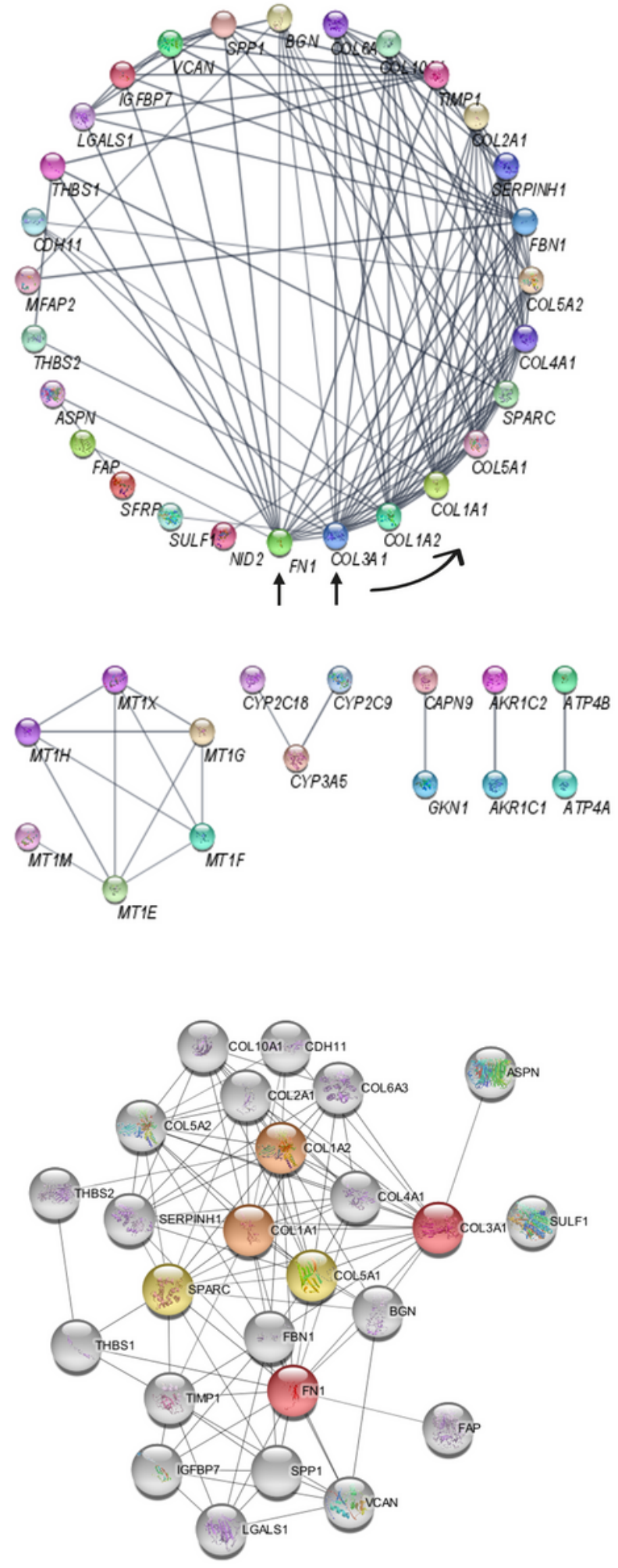

Figure 3 
Protein-protein interaction (PPI) network of 83 differentially expresses genes in gastric cancer. A PPI network graph constructed in STRING. (Line colors indicate the type of interaction evidence; edges indicate functional and physical protein associations. Minimum required interaction score: high confidence (0.7). Disconnected nodes are hidden in the network). B Degree sorted circular network graph constructed in Cytoscape. Black arrows show the two genes with the highest degree (16) in the network. The curved arrow shows the direction of the decrement in node degrees. $\mathbf{C}$ Modules in the protein-protein interaction network of 83 differentially expressed genes in gastric cancer. Module analysis was performed using the MCODE tool in Cytoscape. The scores, number of nodes, and edges for each module are listed in the figure. D Neighbors of the six hub genes COL1A1 (orange), COL1A2 (orange), COL3A1 (red), COL5A1 (yellow), FN1(red), and SPARC (yellow). The network was constructed using the Cytohubba tool in Cytoscape. (Color grade from red to yellow indicate the descending order of node degrees in the network).

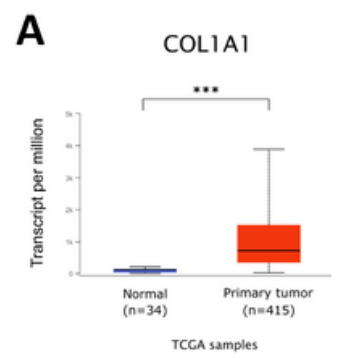

B

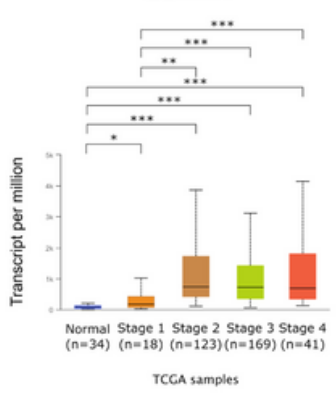

C

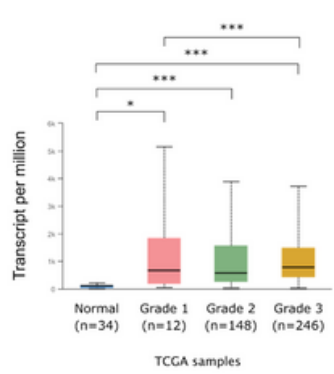

D Colial

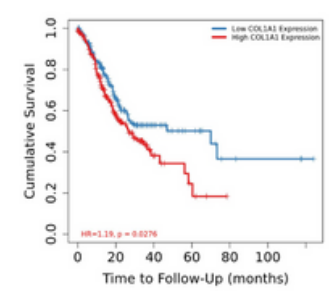

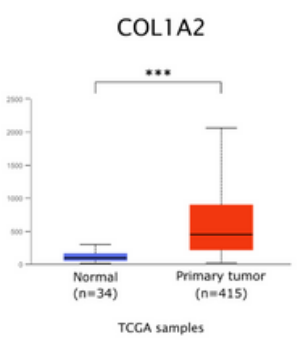

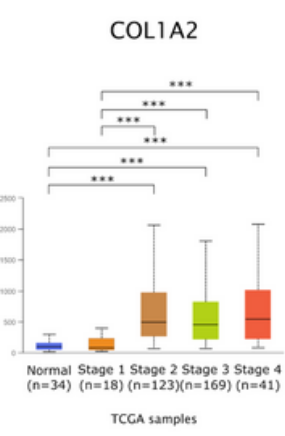

COL1A2

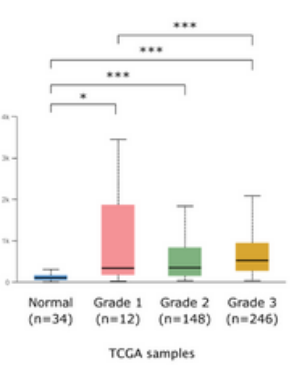

COL1A2

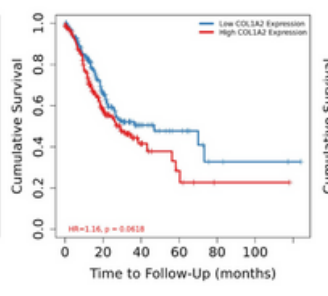

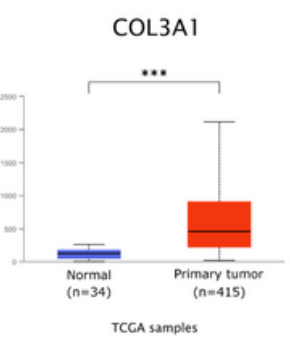

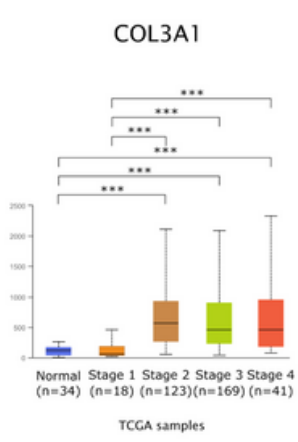

COL3A1

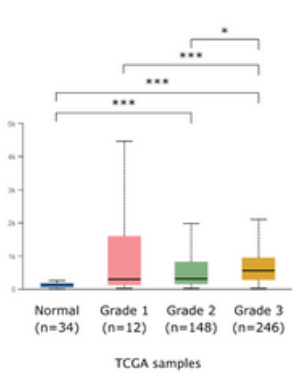

COL3A1

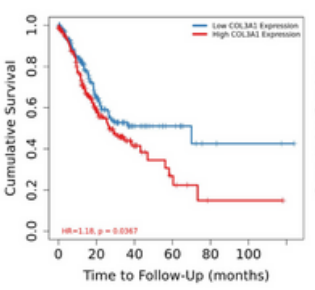

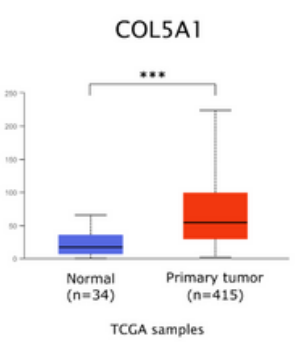

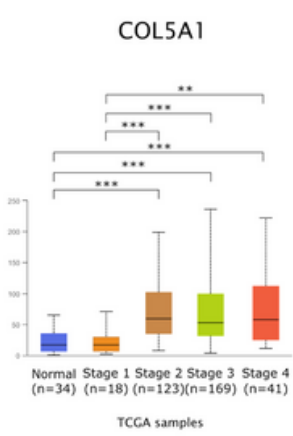

COL5A1

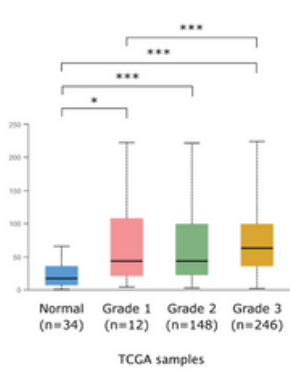

COL5AI

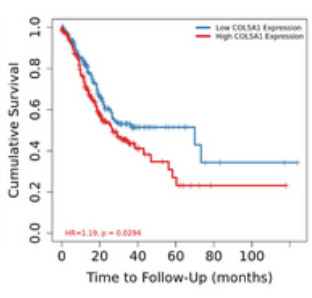

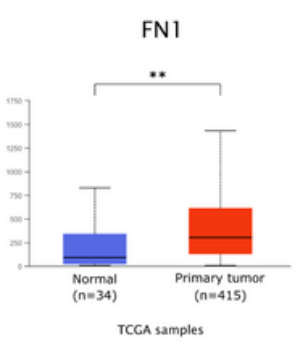

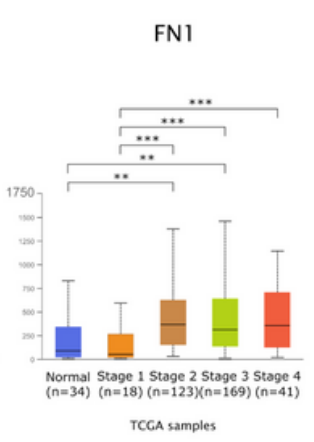

FN1

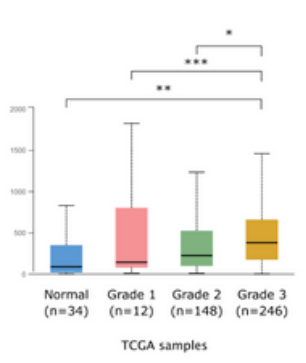

FN1

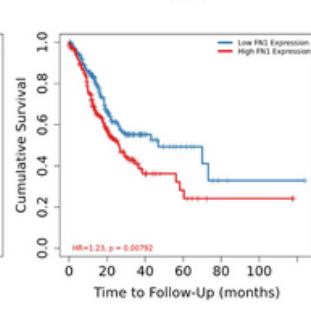

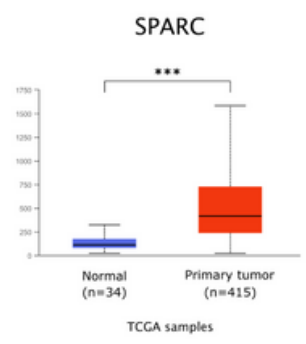

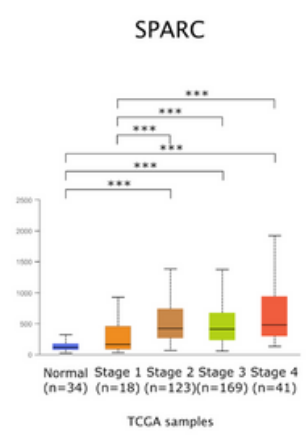

TCCA samples

SPARC

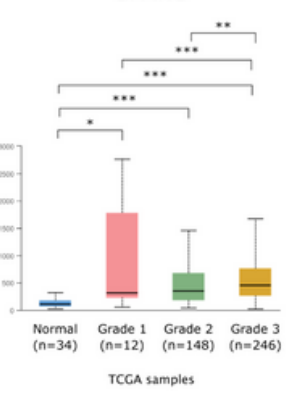

SPARC

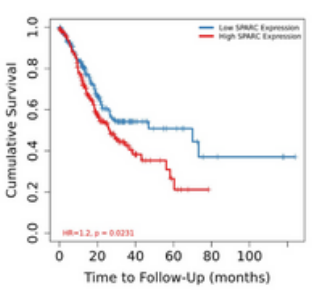




\section{Figure 4}

Differential expression and prognostic significance of the six key genes in gastric cancer. A Differential expression of hub genes in normal tissues vs. primary stomach adenocarcinoma tissues. B Differential expression of the hub genes by tumor stage in primary stomach adenocarcinoma. C Differential expression of hub genes by tumor grade in stomach adenocarcinoma. D Kaplan-Meier survival analysis of hub genes in stomach adenocarcinoma. TCGA data which include 34 normal tissues, and 415 primary stomach adenocarcinoma tissues were used for analysis ( ${ }^{*}<0.05$, $\left.* \star p<0.01, * \star \star ~ p<0.001\right) .(A-C)$ Analysis was performed on the UALCAN. (D) Analysis was performed on TIMER 2.0.
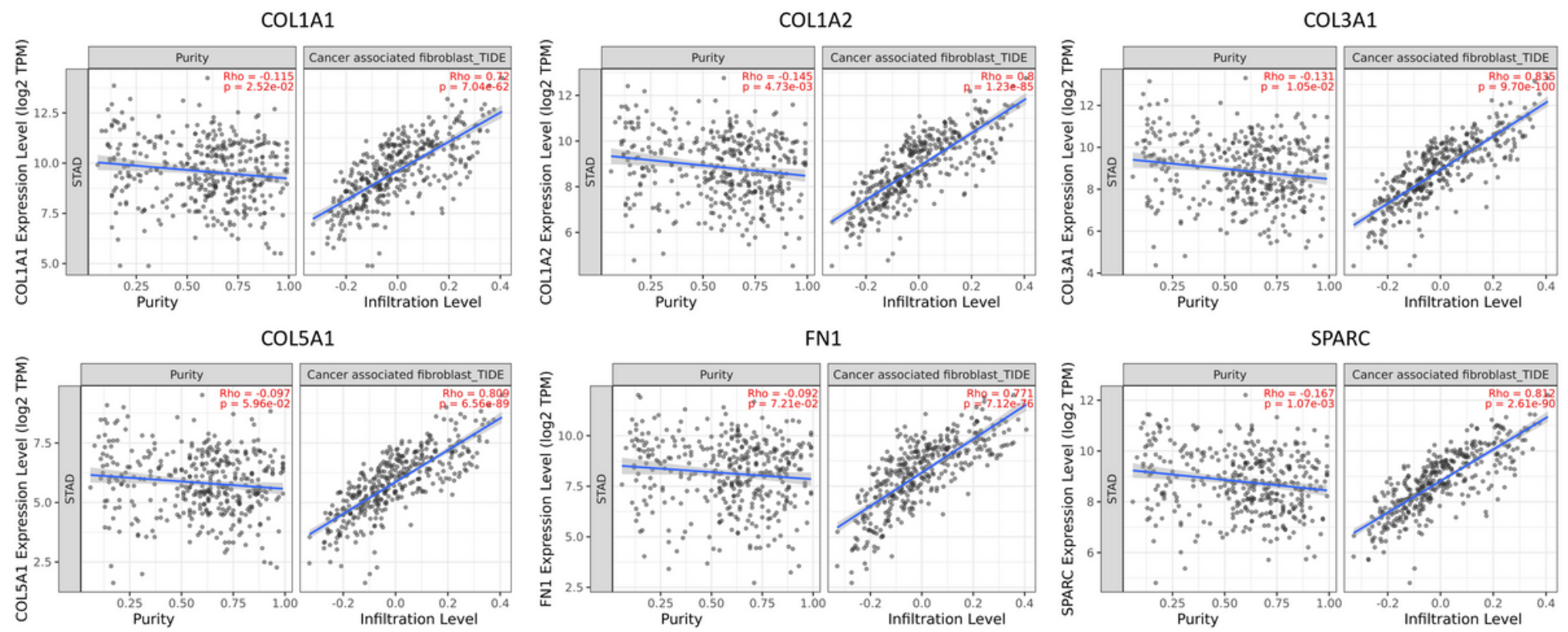

\section{Figure 5}

Correlation of the six key genes with the cancer-associated fibroblast infiltration in stomach adenocarcinoma. TIDE algorithm in TIMER2.0. was used to infer the cancer-associated fibroblast infiltration levels in TCGA stomach adenocarcinoma samples. 

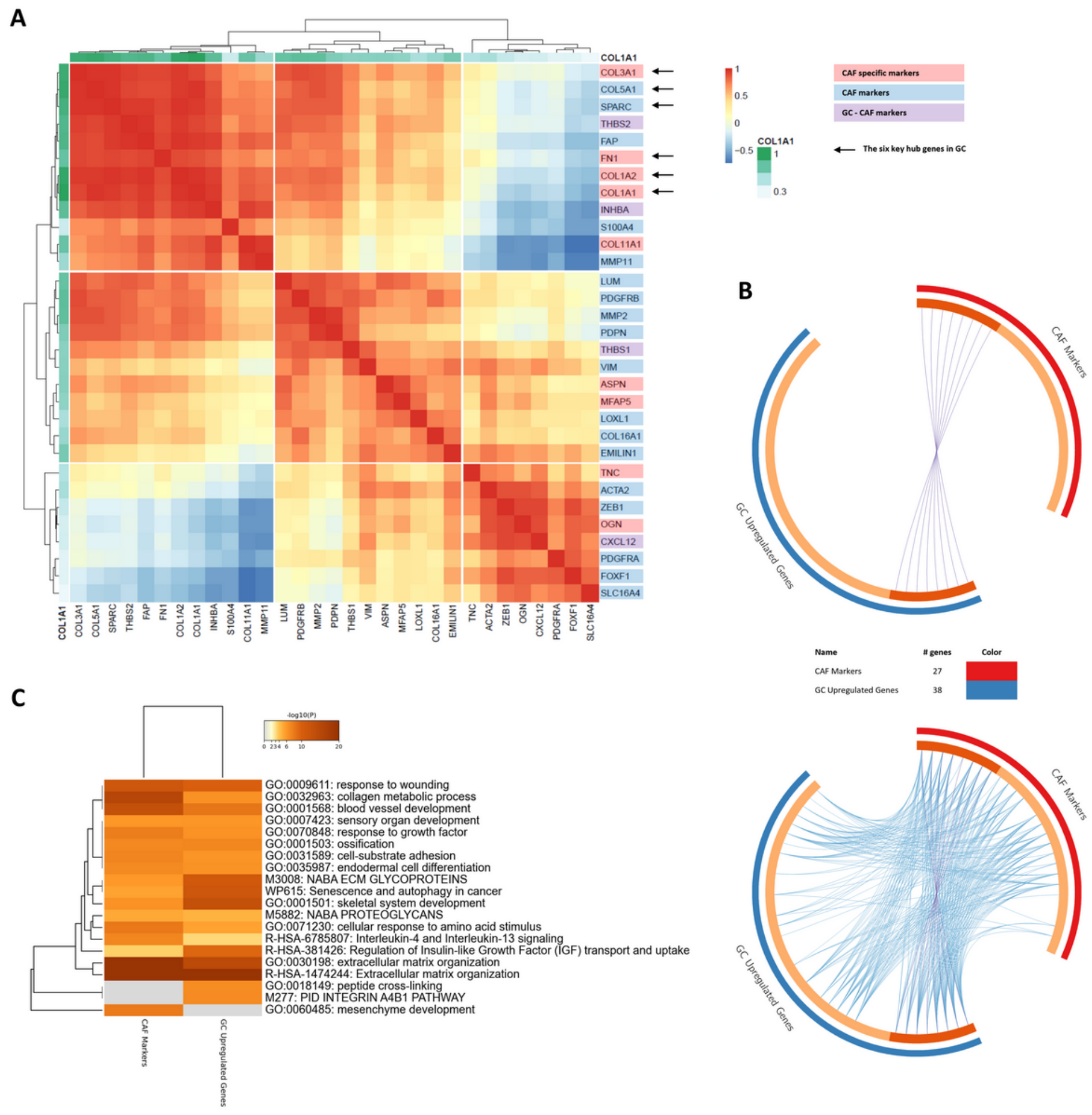

Figure 6

Correlation of the upregulated genes in gastric cancer with the cancer-associated fibroblast markers. A Correlation heatmap for the cancer-associated fibroblast (CAF) markers and the six key genes in gastric cancer (GC). Correlation data was extracted from TIMER2.0 using the "gene correlation" module. Heatmap was created in imageGP using hierarchical clustering. The six key genes in GC are shown with black arrows. Genes are classified as CAF-specific markers(red), CAF markers (blue), and GC-CAF markers (CAF markers identified in GC) (lilac). B The circos plots show how genes from the CAF markers (red outer 
arc) and upregulated genes in GC (blue outer arc) lists overlap. On the inside, each arc represents a gene list, where each gene has a spot on the arc. Dark orange color represents the genes that appear in both lists and light orange color represents genes that are unique to a single gene list. Purple lines (upper circos plot) link the same genes that are shared by the two lists. Blue lines (lower circos plot) link the different genes which fall into the same ontology term. C Enriched ontology clusters for (GO/KEGG terms, canonical pathways) for CAF markers and Upregulated genes in GC. (B-C) Analysis was performed on Metascape.org. 
A

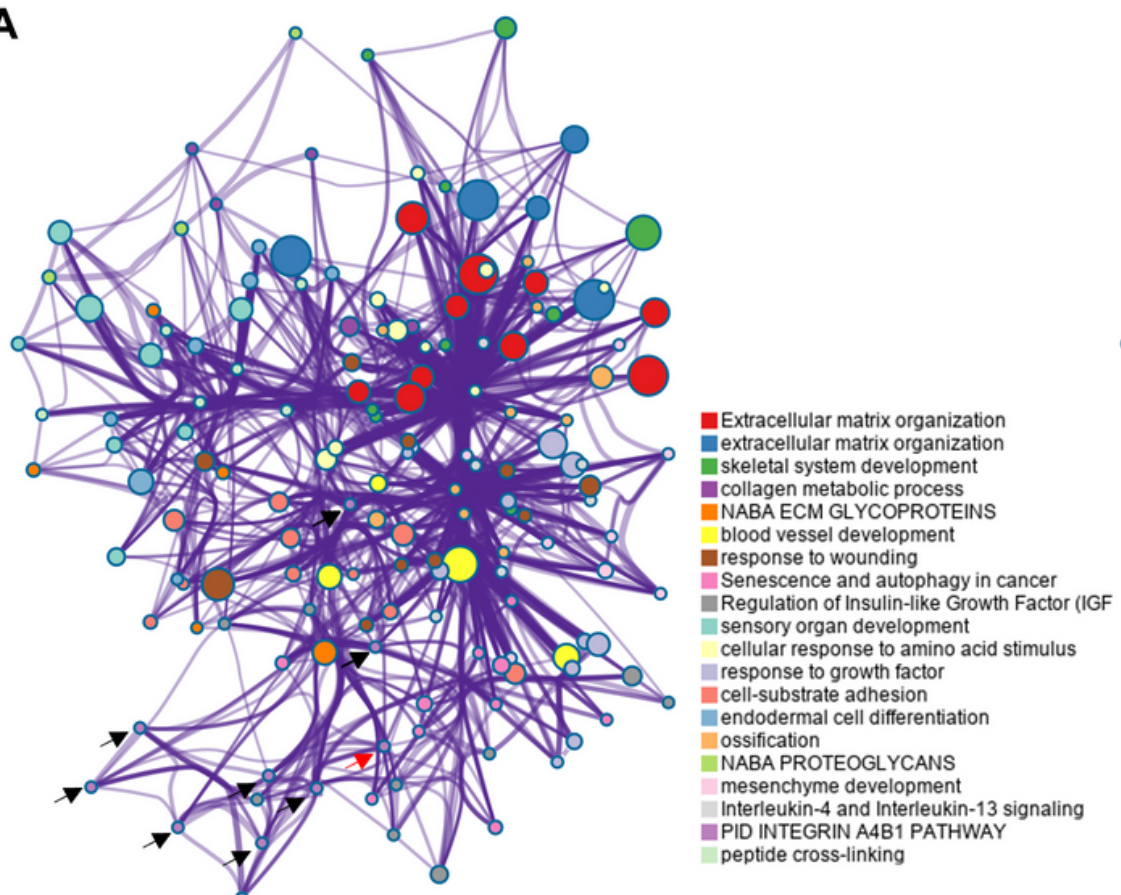

B

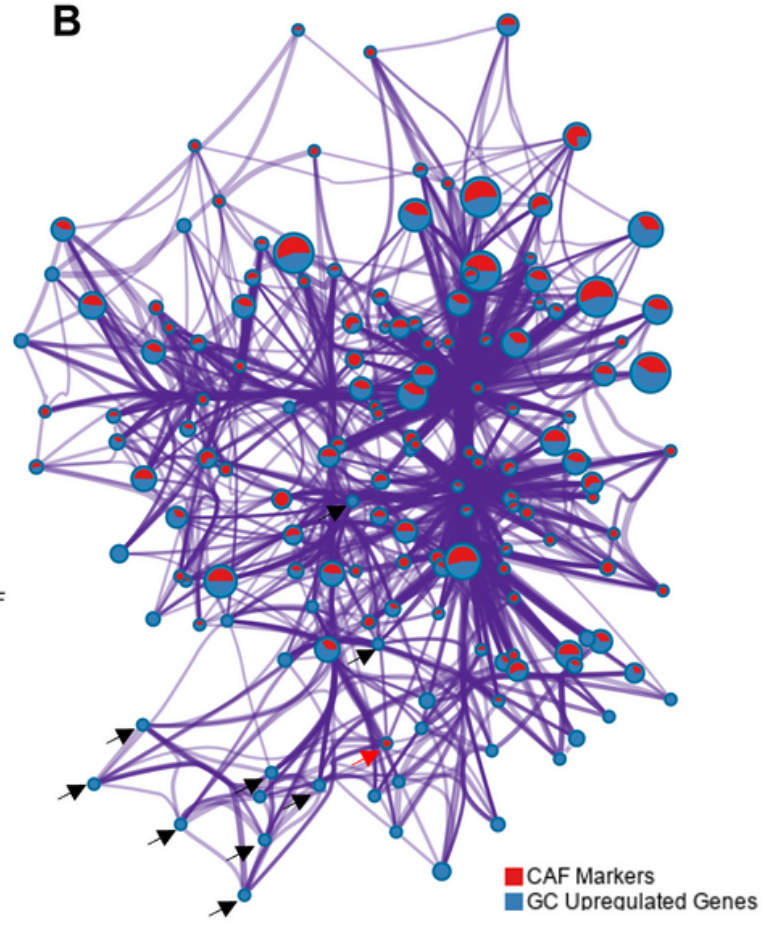

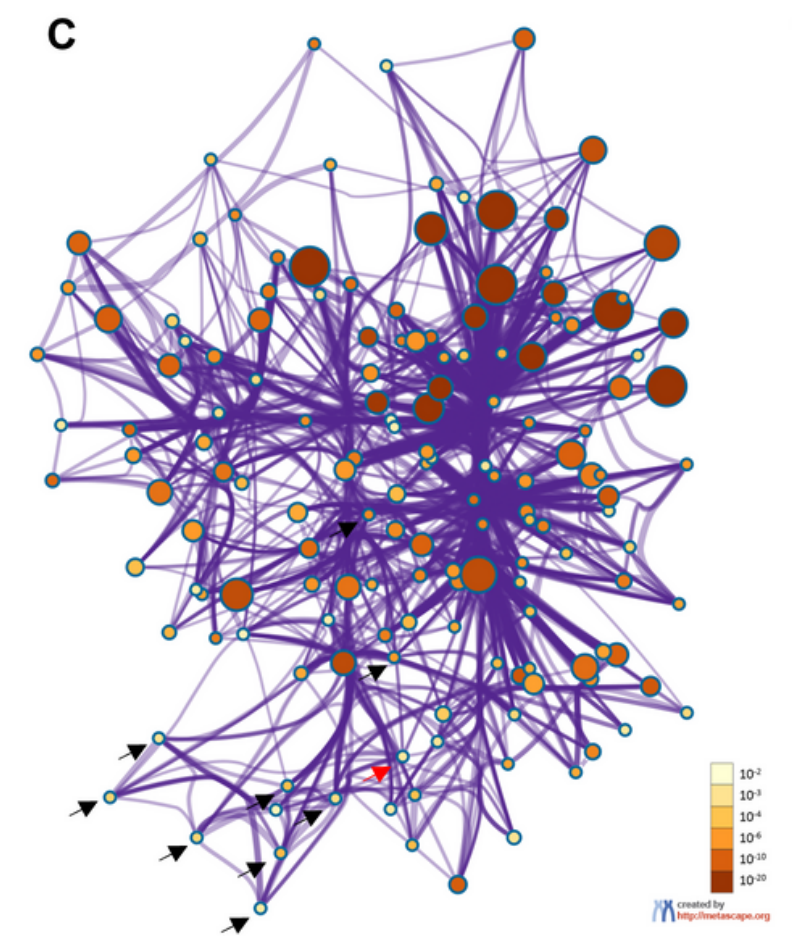

\section{Figure 7}

Enriched ontology clusters for the cancer-associated fibroblast markers and upregulated genes in gastric cancer. A Network representation of enriched terms for a combined list of the cancer-associated fibroblast (CAF) markers and upregulated genes in gastric cancer (GC). Each term is represented by a circle node, where its size is proportional to the number of the input genes that fall into that term and its color represents its cluster identity. B Representation of the same network nodes in A as pies. Each pie sector is proportional to the number of hits originating from the CAF markers list (red) and upregulated genes in GC (blue). C Representation of the same network nodes in A colored by p-value, as shown in the color scale. (A-C) The black arrows show the nodes that fall into the Integrin a $4 \beta 1$ pathway in GC upregulated 
genes list. Only one node that falls into the Integrin a4 $\beta 1$ pathway shown with the red arrow was common in both the CAF markers list and the GC upregulated genes list. The data was analyzed, and the network layouts were prepared in Metascape.org.

A

Gene expression

Z-score : increased risk $(p<0.05,2>0)$

Z-score : decreased risk $(p<0.05, z<0)$

$凶 p>0.05$

- $\mathrm{p} \leq 0.05$

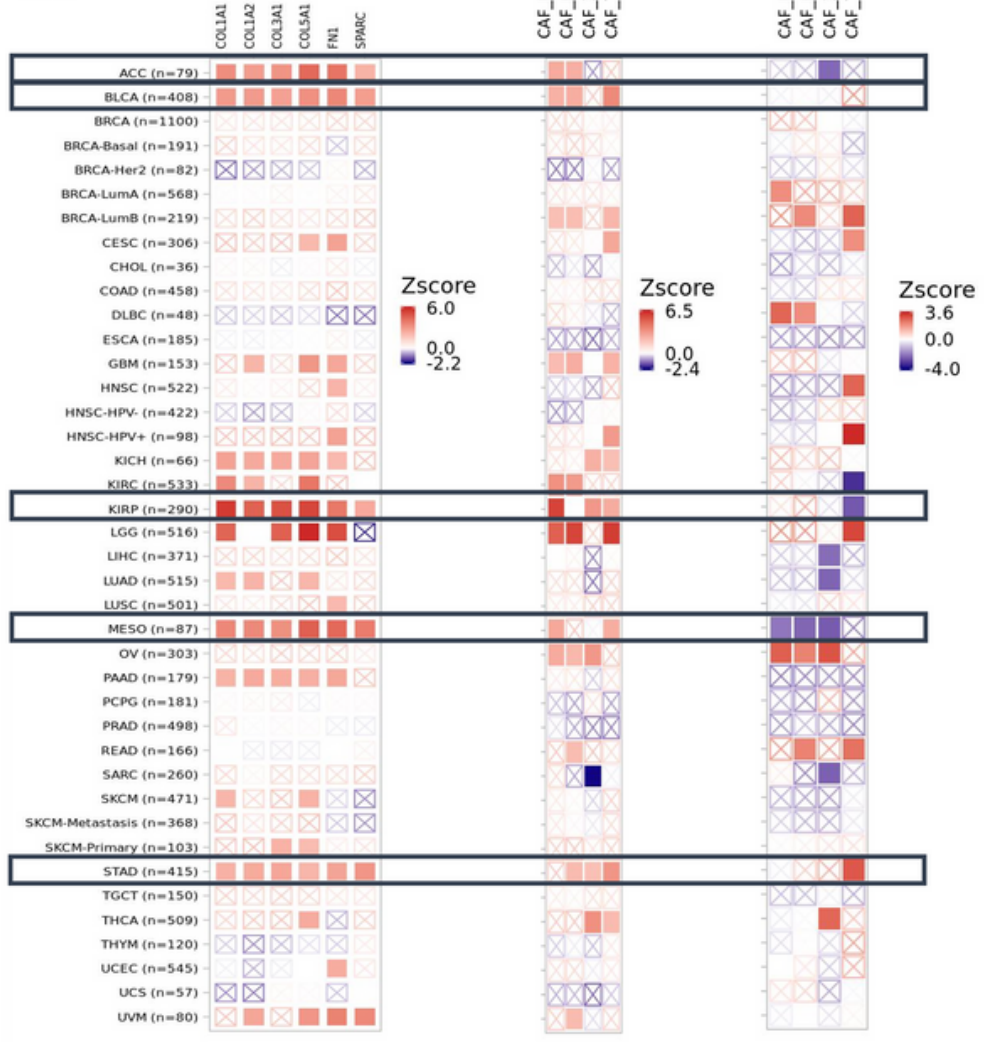

$\mathbf{F}$

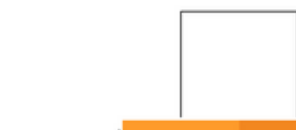

(2)
B

CAF+

紊
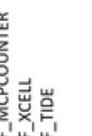

A1\&

荧

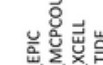

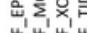

GO:0001503: ossification

GO:00001568: blood vessel development

$\mathrm{GO}: 0071560$ : cellular response to transfor
$\mathrm{GO} 0001501$ : skeletal system developme

GO:0031589: cell-substrate adhesion

GO:0030198: extracellular matrix organization

M5884: NABA CORE MATRISOME

GO:0018149: peptide cross-linking

(n)

R-HSA-8957275: Post-translational protein phosphorylation

GO:0001654: eye development

WP3967: miR-509-3p alteration of YAP1/ECM axis

WP615: Senescence and autophagy in cancer

WP474: Endochondral ossification

GO:0051346: negative regulation of hydrolase activity

E
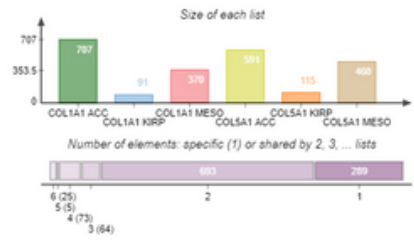

E
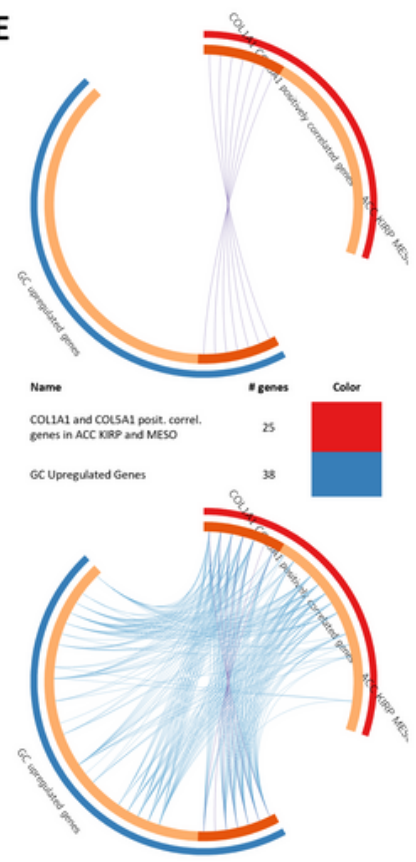

GO:0090100: positive regulation of transmembrane receptor protein serine/threonine kinase signaling pathway

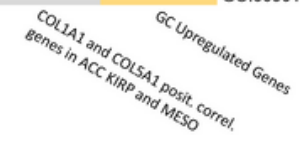

Figure 8 
Prognostic impact of the six key genes and the cancer-associated fibroblast infiltration in cancer. Heatmap of risk scores for $\mathbf{A}$ the expression of COL1A1, COL1A2, COL3A1, COL5A1, FN1 and SPARC, B the cancer-associated fibroblast (CAF) infiltration and, $\mathrm{C}$ the expression of COL $1 A 1$ and $C O L 5 A 1$ plus CAF infiltration in different cancers at TCGA dataset. $(A-C)$ z-score $>0(p<0.05)$ indicates increased risk and zscore $<0$ ( $p<0.05)$ indicates decreased risk. ACC: adrenocortical carcinoma, BLCA: bladder urothelial carcinoma, KIRP: kidney renal papillary cell carcinoma, MESO: mesothelioma, and STAD: stomach adenocarcinoma. Data was extracted from TIMER 2.0. D Venn analysis of genes highly correlated $(r \geq 0.5)$ with COL $1 A 1$ and COL5A1 in TCGA samples of ACC, KIRP, and MESO. E The circos plots that show how genes from the input gene lists- 25 common genes detected by Venn analysis at (D) (red outer circle) vs upregulated genes in gastric cancer (blue outer circle)- overlap. The dark orange color at the inner circle represents the genes that appear in both lists and the light orange color represents genes that are unique to that gene list. Purple lines (upper circos plot) link the same genes that are shared by the two lists. Blue lines (lower circos plot) link the different genes which fall into the same ontology term. $\mathbf{F}$ Enriched ontology clusters (GO/KEGG terms, canonical pathways) for 25 common genes correlated with COL 1A1 and COL5A1 in ACC, KIRP, and MESO vs upregulated genes in GC. (E-F) Analysis was performed on Metascape.org. For a full list of TCGA cancer type abbreviations please refer to https://gdc.cancer.gov/resources-tcga-users/tcga-code-tables/tcga-study-abbreviations.

A

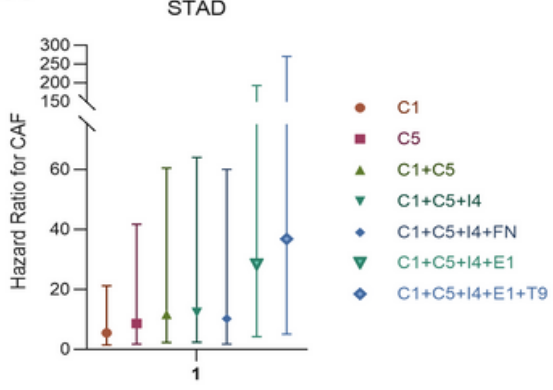

D

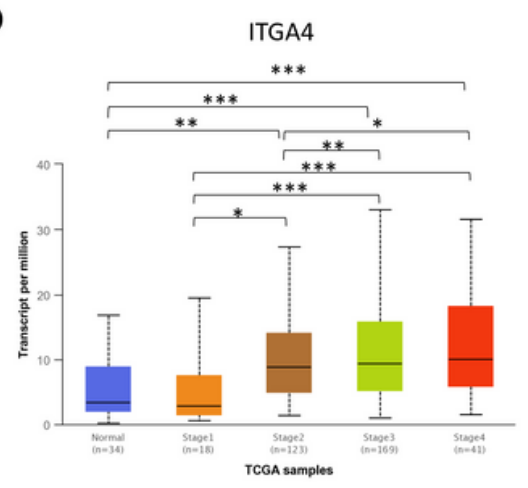

B

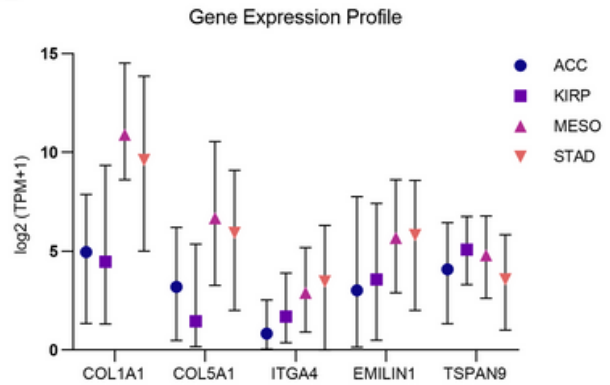

$\mathrm{E}$

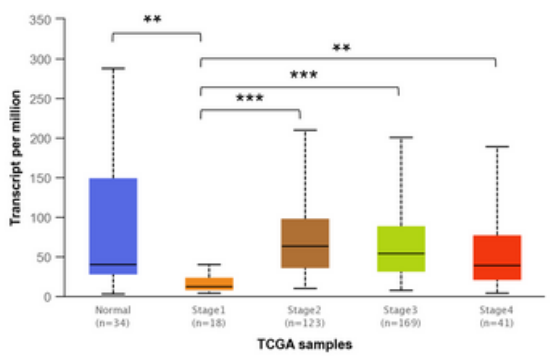

C

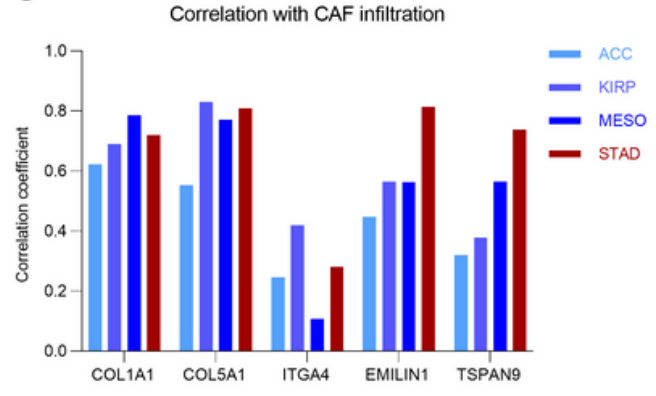

$\mathbf{F}$

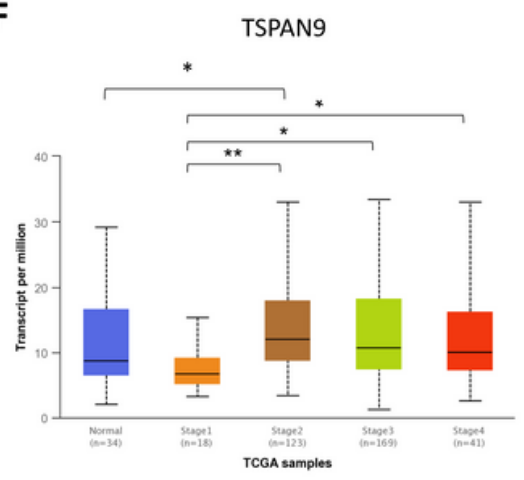

Figure 9

Prognostic impact and the expression profile of the cancer-associated fibroblast signature genes. A Hazard ratios of CAF infiltration for different gene expression profiles in STAD. C1: COL 1A1, C5: COL5A1, 
14: ITGA4, E1: EMILIN1, F1: FN1 and T9: TSPAN9. Bars indicate 95\% confidence intervals. Data was extracted from TIMER2.0. B Gene expression profile for COL1A1, COL5A1, ITGA4, EMILIN1, and TSPAN9 in ACC, KIRP, MESO, and STAD. Symbols show the medians for transcripts per million (TPM) in each cancer at TCGA dataset. Data was extracted from UALCAN. C Correlation between CAF infiltration and the expression of COL1A1, COL5A1, ITGA4, EMILIN1, and TSPAN9 in ACC, KIRP, MESO, and STAD. Data was extracted from TIMER2.0. using the TIDE algorithm. Differential expression of D ITGA4, E EMILIN1, and $\mathbf{F}$ TSPAN9 by tumor stage in primary STAD. The TCGA data, which includes 34 normal tissues and 415 primary stomach adenocarcinoma tissues, was used for analysis. Analysis was performed on UALCAN (unpaired t-test, ${ }^{\star} p<0.05, * \star p<0.01, * \star \star p<0.001$ ). (ACC: adrenocortical carcinoma, BLCA: bladder urothelial carcinoma, KIRP: kidney renal papillary cell carcinoma, MESO: mesothelioma, and STAD: stomach adenocarcinoma).
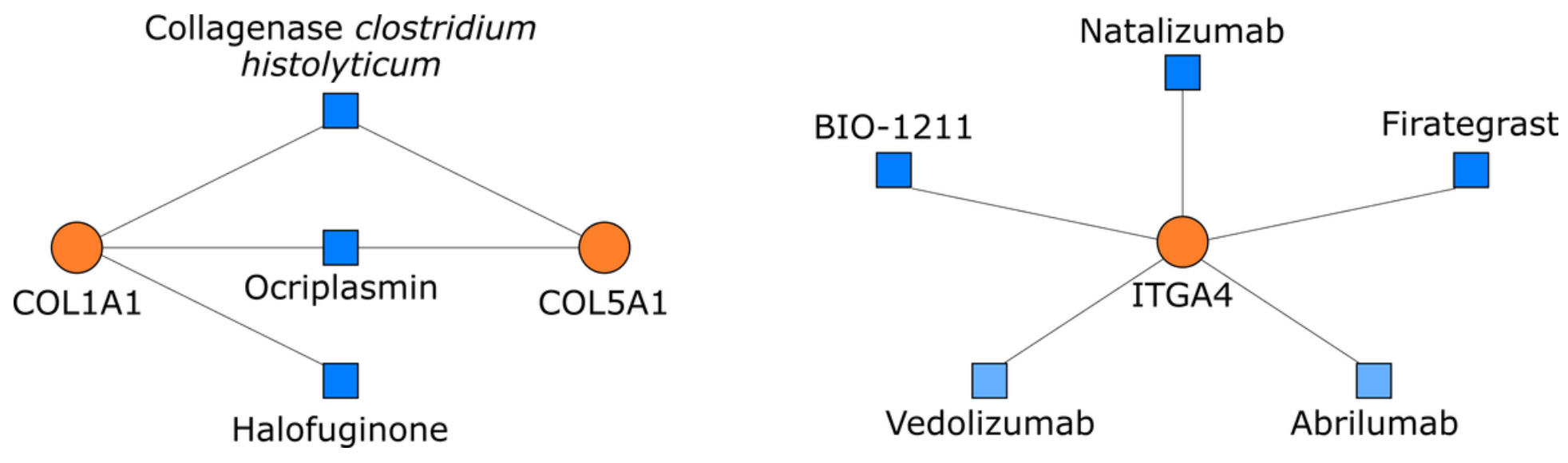

Figure 10

Currently available drugs that target COL1A1, COL5A1, and ITGA4. Drugs (shown with blue squares) listed in DrugBank and Drug-gene interaction database that target COL1A1, COL5A1, or ITGA4 (shown with orange circles). Among the ITGA4 targeting drugs, the action of abrilumab, and vedolizumab, which are shown with light blue, are specific to integrin a4 $\beta 7$ rather than integrin a4 $\beta 1$. Natalizumab, firategrast, and BIO-1211 target integrin a4.

\section{Supplementary Files}

This is a list of supplementary files associated with this preprint. Click to download.

- AdditionalFile1.docx

- AdditionalFile2.pdf 